\title{
Hydrogeologische Notizen
}

Online publiziert: 8. November 2021

(c) Springer-Verlag GmbH Deutschland, ein Teil von Springer Nature 2021

\section{Grundwasser in eigener Sache}

Die Redaktion behält sich vor, eingereichte Beiträge zu kürzen oder redaktionell zu verändern.

\section{Redaktionsschluss für Beiträge zu den Hydrogeologischen Notizen}

Liebe Kollegen und Kolleginnen, in den hydrogeologischen Notizen bieten wir aktuelle und für einen großen Leserkreis interessante Mitteilungen. Damit das auch weiterhin so bleibt, freuen wir uns auch auf Ihre Beiträge. Sie können zu allen Rubriken unseres Nachrichtenteils Beiträge einreichen.

Damit wir Ihre Beiträge auch zeitnah drucken können, beachten Sie bitte unsere Termine zum Redaktionsschluss:

- Heft 1/22: 17.12.2021

- Heft 2/22: 28.02.2022

- Heft 3/22: 10.06.2022

- Heft 4/22: 02.09.2022

Ihre Beiträge senden Sie bitte an: Patricia Schüll

E-Mail: p.schuell@gmx.de

Vielen Dank für Ihre Unterstützung!

Die Redaktion

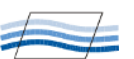

\section{Fachsektion Hydrogeologie} (FH-DGGV)

\section{Einladung zur Mitglieder- versammlung 2022 der Fachsektion Hydrogeologie e.V. in der DGGV e.V.}

Sehr geehrtes Mitglied der Fachsektion Hydrogeologie,

hiermit laden wir Sie herzlich zur Mitgliederversammlung anlässlich der FH-DGGV-Tagung in Jena ein. Ort: FSU Jena, Carl-Zeiss-Str. 3, HS1 Zeit: Donnerstag, 24.03.2022, 17.30 Uhr

\section{Tagesordnung}

TOP 1: Begrüßung

TOP 2: Ehrungen

TOP 3: Bericht des Vorstands

TOP 4: Bericht der Geschäftsstellenleiterinnen

TOP 5a: Bericht der Schatzmeisterin

TOP 5b: Bericht über die Stiftung Grundwasser

TOP 6: Bericht der Kassenprüfer

TOP 7: Entlastung des Vorstands

TOP 8: Berichte der Sprecher der Arbeitskreise

TOP 9: Benennung des Wahlvorstands TOP 10: Neuwahl des/r 1. Vorsitzenden TOP 11: Neuwahl des/r Schriftführer/ in
TOP 12: Wahl von fünf Beiratsmitgliedern

TOP 13: Nächste Tagungen der FHDGGV

TOP 14: Verschiedenes

Für die neu zu wählenden Ämter unterbreitet der Vorstand folgende Wahlvorschläge:

1. Vorsitzender:

Prof. Dr. Nico Goldscheider, Karlsruher Institut für Technologie

Schriftführer:

Dr. Andreas Musolff, Helmholtz-Zentrum für Umweltforschung $\mathrm{GmbH}$, Leipzig

Beiratsmitglieder:

Peter Börke, Sächsisches Landesamt für Umwelt Landwirtschaft und Geologie Dresden

Victoria Burke, Universität Oldenburg Bernhard Keim, Kobus und Partner Stuttgart

Tobias Licha, Ruhr-Universität Bochum

Dirk Radny, Bundesamt für Gewässerkunde Koblenz

Es können weitere Wahlvorschläge von jedem Mitglied eingereicht werden. 


\section{Hinweis}

Laut Satzung kann das Stimmrecht nur auf der Mitgliederversammlung ausgeübt werden. Mitglieder, die nicht an der Versammlung teilnehmen können, haben jedoch die Möglichkeit, sich mit schriftlicher Vollmacht durch ein anderes Mitglied vertreten zu lassen.

gez. Prof. Dr. T. Scheytt Vorsitzender der FH-DGGV gez. Dr. A. Musolff Schriftführer der FH-DGGV

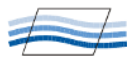

\section{Mitgliederversammlung 2022 - Es stellen sich zur Wahl}

\section{Kandidat Erster Vorsitzender}

Prof. Dr. Nico Goldscheider ist Professor für Hydrogeologie am Karlsruher Institut für Technologie (KIT). Er hat in Karlsruhe Geologie studiert und in der Hydrogeologie promoviert. Von 2002 bis 2010 war er Oberassistent an der Universität Neuchâtel (Schweiz) und anschließend Professor für $\mathrm{Hy}$ drogeologie und Geothermie an der TU München, bis er 2011 ans KIT berufen wurde. Seine Forschungsschwerpunkte sind Karst und alpine Hydrogeologie, Grundwasserqualität und ökologischer Grundwasserschutz. 2003 hat er die IAH-Kommission für Grundwasser und Klimawandel mitbegründet und mitgeleitet; von 2009 bis 2017 war er Vorsitzender der IAHKarstkommission und leitet aktuell ein europäisches Verbundprojekt über Mediterrane Karstwasserressourcen. Er war Assoziierter Editor mehrerer Fachzeitschriften, hat ein Lehrbuch und über 100 Papers publiziert. Für die FH-DGGV hat er an mehreren Fortbildungen mitgewirkt, ein Doppel-Themenheft über Karsthydrogeologie als Gasteditor zusammengestellt und von 2018 bis 2021 als Editor von Grundwasser gedient. Als Vorsitzen- der möchte er gemeinsam mit dem FH-DGGV-Team und allen Mitgliedern den erfolgreichen Kurs der letzten Jahre fortsetzen und zu einer Stärkung von Grundwasser-Themen in öffentlichen Diskussionen beitragen.

\section{Kandidat Schriftführer}

Dr. Andreas Musolff studierte von 1995 bis 2001 an der Universität Greifswald Geologie mit Vertiefung ,Hydrogeologie“. Seine Diplomarbeit zur GIS-basierten Abschätzung von Grundwasserneubildungsraten erhielt 2002 den Preis „Hydrogeologie“ des Arbeitskreises „Ausbildung und Information“ der FH-DGG. Nach dem Abschluss des Studiums war er als Projektmanager für Geoinformationssysteme und für Datenmanagement in der freien Wirtschaft tätig. Von 2006 bis 2009 führte Herr Musolff seine Dissertation am Helmholtz-Zentrum für Umweltforschung (UFZ) in Leipzig durch. Hier forschte er zu zeitlich-räumlicher Variabilität von Mikroschadstoffen in urbanen Grund- und Oberflächenwässern und der Interaktion der verschiedenen urbanen Wasserkompartimente. Seit seiner Promotion 2009 an der Universität in Neuchâtel in der Schweiz ist Herr Musolff als Wissenschaftler am UFZ in Leipzig am Department Hydrogeologie tätig. Hier arbeitet er an der Bewertung und Modellierung von Wasser- und Stoffflüssen in Flusseinzugsgebieten mit besonderem Augenmerk auf Nitrat und gelöstem organischen Kohlenstoff.

\section{Kandidaten und Kandidatinnen für den Beirat}

Dr. Peter Börke studierte von 1989 bis 1994 Grundwasserbewirtschaftung an der TU Dresden und war danach im Dresdner Grundwasserforschungszentrum beschäftigt. Nach einem einjährigen Forschungsaufenthalt in den USA kehrte er nach Deutschland zurück und begann eine Tätigkeit am Sächsischen Landesamt für Umwelt und Geologie als Referent für Altlas- tensanierung. Ab 2003 leitete er ein Verbundvorhaben im BMBF-Förderschwerpunkt „KORA“, welches sich mit natürlichen Abbauprozessen im Untergrund (natural attenuation) beschäftigte. Hierbei wurden ein Leitfaden sowie Handlungsempfehlungen für den Umgang mit Teerölen und Gaswerks- und Kokereirückständen entwickelt. Nach Abschluss dieses Vorhabens und einer Promotion mit Thema der passiven Grundwasserprobennahme bei organischen Kontaminanten leitete er am Landesamt bis 2010 den Grundwasserbeobachtungsdienst des Freistaates Sachsen. Dann wechselte er für zwei Jahre an das Staatsministerium für Umwelt und Landwirtschaft (SMUL) und erarbeitete federführend eine neue Grundsatzkonzeption Wasserversorgung für den Freistaat. 2015 wurde er zum Referatsleiter Siedlungswasserwirtschaft, Grundwasser am Landesamt für Umwelt, Landwirtschaft und Geologie berufen. Seine fachlichen Schwerpunkte liegen in der Grundwasserbeobachtung und der Dargebotserkundung für die Trinkwasserversorgung. Er arbeitet in Regelwerksgremien des DVGW mit und leitet den Arbeitskreis Grundwasserbeobachtung der Länder Berlin, Bayern, Brandenburg, Thüringen und Sachsen-Anhalt und Sachsen.

Dr. Victoria Burke studierte zunächst Geologie an der Freien Universität Berlin und ist seit dem Jahre 2010 an der Carl von Ossietzky Universität in Oldenburg tätig. Ihre Forschungsschwerpunkte liegen im Themenkomplex der organischen Spurenstoffe, denen sie sich in erster Linie anhand von laborexperimentellen Methoden nähert. So promovierte sie zum Thema „Redoxsensitivität und Langzeitpersistenz pharmazeutischer Rückstände im Grundwasser“. Zuletzt leitete sie eine Juniorforschergruppe zum Thema „Tierarzneimittel in Boden und Grundwasser", in deren Rahmen das Umweltverhalten verschiedener Tierarzneimittelrückstände auf deren Pfad von der Eintragsquelle über die unge- 
sättigte sowie gesättigte Zone anhand von Labor- und Feldversuchen auf unterschiedlichen Skalen qualitativ und quantitativ untersuchte wurde.

Bernhard Keim hat zwischen 1983 und 1989 an der Universität Stuttgart Bauingenieurwesen studiert. Verschiedene Wasserthemen und Geotechnik bildeten den Studienschwerpunkt. Seit 1995 ist er geschäftsführender Gesellschafter der Ingenieurgesellschaft Prof. Kobus und Partner GmbH mit Sitz in Leinfelden-Echterdingen. Er kann auf ein sehr breites Spektrum an Projekten verweisen. Im Mittelpunkt stehen Grundwasserstudien. Diese umfassen hydrogeologische Erkundungen und Monitoring sowie Modelle. Die Bearbeitung von hydraulischen Projekten in natürlichen Gewässern und technischen Systemen sowie hydrologische Arbeiten runden sein berufliches Spektrum ab. Die Projektarbeit ist meist von einer Interdisziplinären Zusammenarbeit, firmenintern und -extern, und einem intensiven Austausch mit Kunden und Behörden geprägt. Neben diesen Tätigkeiten engagierte sich Bernhard Keim in verschiedenen Arbeitskreisen des DVGW und im AK „Hydrogeologie arider Gebiete" der FH-DGGV. Dieses Engagement würde er gerne als Beirat der FH-DGGV fortsetzen. Das in der Vereinssatzung definierte Verständnis, ein „Forum für Wissenschaft und Praxis in der Hydrogeologie" zu bilden, deckt sich dabei vollständig mit seinen Interessen und seiner Motivation als Kandidat für den Beirat.

Prof. Dr. Tobias Licha studierte Chemie in Leipzig sowie Hydrogeologie in London und promovierte 2003 an der chemisch-geowissenschaftlichen Fakultät in Jena zum Thema „Umweltverhalten von Phenolen“. Von 2003 bis 2019 arbeitete er als Laborleiter und von 2008 bis 2019 als Leiter der AG Hydrochemie am Lehrstuhl für Angewandte Geologie in Göttingen. Die Nutzung organischer Moleküle zur Charakterisierung von Georeservoiren war zentrales Element seiner 2013 abgeschlossenen Habilitation. Seit 2019 ist er an der Ruhr-Universität Bochum Professor für Hydrogeochemie. $\mathrm{Zu}$ seinen Arbeitsschwerpunkten zählen insbesondere die Nutzung und Interpretation organischer Verbindungen zur Identifizierung von Prozessen im Grundwasser und in Georeservoiren.

Dr.-Ing. Dirk Radny studierte Angewandte Geowissenschaften mit Vertiefung Hydrogeologie an der Technischen Universität Berlin, wo er das Studium 2006 als Diplom-Ingenieur abschloss und 2010 promovierte. Bis Februar 2013 war Herr Radny Wissenschaftlicher Assistent im FG Hydrogeologie an der TU Berlin. Im März 2013 wechselte Herr Radny zur Schweizerischen Eawag in die Hydrogeologie-Gruppe von Mario Schirmer. Seine Hauptaufgaben an der Eawag waren Projektkoordination und die Konzeption, Durchführung und Auswertung von umfangreichen Probennahmekampagnen. Zwischen August 2015 und Juli 2016 übernahm er die kommissarische Leitung der Hydrogeologie-Gruppe während des Sabbaticals von Herrn Schirmer. Seit Oktober 2017 ist Herr Radny an der Bundesanstalt für Gewässerkunde fachlicher Leiter im Aufgabenbereich „Hydrogeologie“ und stellvertretender Referatsleiter des Referates „Grundsatzfragen der qualitativen Gewässerkunde“. $\mathrm{Zu}$ seinen Aufgaben gehört u. a. die fachlich-wissenschaftliche Begleitung des BMVI und der WSV bei allen Fragen zur Grundwasserbeschaffenheit entlang der Bundeswasserstrassen. Die fachlichen Interessensschwerpunkte von Herrn Radny liegen in den Bereichen ,feldorientierte Hydrogeologie" und Altlastenbearbeitung.

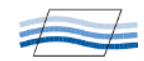

\section{Neue Geschäftsstellenleitung}

Liebe Mitglieder der FH-DGGV, wie die meisten von Ihnen sicherlich schon gelesen haben, bekommt die Geschäftsstelle der FH-DGGV eine neue Anschrift und eine neue Leitung.

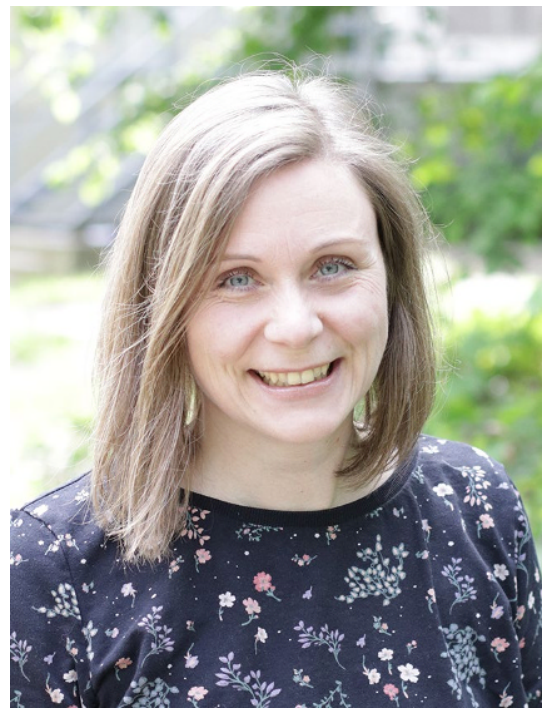

Abb. 1 Maike Rüsgen (Foto: N. Houdelet)

Damit Sie über die neue Geschäftsstellenleiterin mehr wissen als nur Namen und Funktion, möchte ich mich Ihnen mit ein paar Sätzen vorstellen (Abb. 1).

Ich habe in Bonn und Karlsruhe Geologie studiert und über die Nutzung von künstlicher Intelligenz in der oberflächennahen Geothermie promoviert. Danach bin ich der Geologie für einige Jahre untreu geworden und habe verschiedene Stationen im Wissens- und Technologietransfer, vor allem im Umfeld der Bauchemie durchlaufen. Ich war bei einer Ausgründung als Projektmanagerin tätig, habe als wissenschaftliche Mitarbeiterin verschiedene Projekte im Innovationsmanagement bearbeitet und zuletzt in einem Helmholtz Innovation Lab am Karlsruher Institut für Technologie gearbeitet.

Nun freue ich mich sehr, dass ich mit der Tätigkeit in der Geschäftsstelle der FH-DGGV wieder in die Hydrogeologie und ihre Community zurückkehre. Ich übernehme von Ruth KaufmannKnoke eine hervorragend strukturierte und gut organisierte Geschäftsstelle. Diese möchte ich in bewährter Form weiterführen und gleichzeitig gemeinsam mit dem Vorstand die FH-DGGV weiterentwickeln. Auch in der FHDGGV sind Themen wie Digitalisie- 
rung, Wissensmanagement oder neue Kommunikationsformate präsent und wollen vorangebracht werden. Viele Erfahrungen der letzten Jahre im Wissens- und Technologietransfer werde ich dabei sicherlich nutzen können.

Mit Menschen im Austausch zu sein - fachlich wie persönlich - ist normalerweise Herausforderung und Bereicherung zugleich. Privat genieBe ich gesellige Runden bei gutem Essen oder feinem Kaffee. Und beruflich freue ich mich darauf, mit Ihnen über die Hydrogeologie und die Herausforderungen, die beispielsweise Ressourcenverknappung, Klimawandel und Energiewende für unseren Fachbereich mit sich bringen, ins Gespräch zu kommen. Und auch ganz besonders auf den Austausch über die FH-DGGV - wie sie heute ist und wie sie in $\mathrm{Zu}$ kunft sein sollte. Damit das gelingen kann, hier die neuen Kontaktdaten der Geschäftsstelle in Karlsruhe:

Geschäftsstelle der FH-DGGV e.V. c/o macio Raumstation

Emmy-Noether-Str. 17

76131 Karlsruhe

Tel: +49 72148070470

E-Mail: maike.ruesgen@fh-dggv.de

Viele Grüße

Maike Rüsgen

\section{FH-DGGV-Tagung: Grundwasser - Klima - Gesellschaft, 23.-26.03.2022 in Jena}

Vom 23. bis 26. März 2022 ist die Friedrich-Schiller-Universität Jena und damit zum ersten Mal Thüringen der Gastgeber der 28. FH-DGGV Tagung.

Die FH-DGGV-Tagung 2022 „Grundwasser-Klima-Gesellschaft" stellt die gesellschaftlichen Herausforderungen um die Ressource Grundwasser, deren Gefährdungen und Nutzungen unter den Bedingungen des
Globalen Wandels in den Fokus. Im Zuge der Energiewende tritt der geologische Untergrund zunehmend als Energie-Speicherort, aber auch als direkte oder indirekte Energiequelle im Rahmen z.B. der Geothermie oder der Gewinnung strategischer Metalle in den Vordergrund. Dies umfasst auch die Nutzung des Untergrundes als Deponiespeicherraum speziell im Rahmen der Anstrengungen zur Standortfindung eines Endlagers hochradioaktiver Abfälle. Die Grundwasserqualität in all ihren Aspekten muss trotz all dieser Bedarfe geschützt werden und nachhaltig nutzbar sein. Vor dem Hintergrund der zusätzlichen Veränderungen durch den Klimawandel mit zunehmenden Extremen wie Hitzewellen und Dürren verlangt die nachhaltige Grundwasser-Nutzung ein angepasstes und vorausschauendes Management der Landnutzung. Dies erfordert einen interdisziplinären und synoptischen Gesamtansatz, der nur durch die Integration der verschiedenen wissenschaftlichen Disziplinen erfüllbar sein wird. Das Institut für Geowissenschaften der FSU Jena hat auf diese Herausforderung mit einer Schwerpunktsetzung in den Studiengängen Geowissenschaften, Umwelt- und Georessourcenmanagement (UGM) und insbesondere in den Biogeowissenschaften reagiert.

Im Rahmen der FH-DGGV-Tagung 2022 wollen wir mit Vertretern aus Forschung, Behörden, Industrie und Verbänden einem Gedankenaustausch jenseits des eigenen Kerngebiets Freiraum geben. In insgesamt 16 Sessions werden dazu Aspekte u. a. im Bereich von sedimentären Grundwassersystemen, ariden Gebieten, der Grundwasserversalzung, künstlicher Grundwasseranreicherung, Bergbaugebieten und Modellierungsansätze unter Verwendung künstlicher Intelligenz aufgegriffen. Das Vortrags- und Posterprogramm wird durch Fortbildungsveranstaltungen und Exkursionen komplettiert. Wir freuen uns auf Ihre Beiträge und einen spannenden interdisziplinären Gedankenaustausch in Jena.

\section{Sessions}

- Grundwasserressourcen und Ökosysteme in Karstregionen

- Identifizierung landwirtschaftlich bedingter Indikatoren im Grundwasser

- Grundwasserversalzung - Ursachen, Herausforderungen und Ausblicke

- Hydrogeologie arider Gebiete

- Hydrogeologie in der Praxis I Fallbeispiele der Staatlichen Geologischen Dienste

- Hydrogeologie in der Praxis II

- Künstliche Intelligenz in der Hydrogeologie

- Temperaturänderungen im Grundwasser: Ursachen, Prozesse und Auswirkungen

- Bergbau und Grundwasser

- Geotechnische Anwendungen in Grundwassersystemen

- Verweilzeiterkundung in Grundwasserleitern mittels Tracern und Grundwasseraltern

- Dürreperioden - Herausforderungen für die zukünftige Grundwasserbewirtschaftung

- Artificial and natural groundwater recharge (IAH Session)

- Hydrogeologie und Standortauswahl für ein tiefengeologisches Endlager (mit DGGV)

- Grundwasserqualitätsentwicklung - Erkenntnisse aus Langzeitstudien in der Kritischen Zone

- Freie Themen

\section{Vorläufiges Programm}

\subsubsection{2}

- Fortbildungsveranstaltungen I und II

- Exkursionen I

- Forum Junge Hydrogeologen

- Jobbörse

- Icebreaker-Party 
24.03.2022

- Vortrags- und Postersessions

- Mitgliederversammlung der FHDGGV

- Gesellschaftsabend

25.03.2022

- Vortrags- und Postersessions

- Mitgliederversammlung der IAH

- Abendvortrag

26.03.2022

- Vortrags- und Postersessions

- Exkursionen II bis V

\section{Fortbildungsveranstaltungen}

Termin: 23.03.2022, 10.00-17.00 Uhr

I. Bohrloch-Geophysik in der Praxis

- vom Messwert zum Ergebnis

Dozenten: Dr. Thomas Wonik, Dr. Christian Zeeden, Katharina Leu, Thomas Grelle; Leibniz-Institut für Angewandte Geophysik (LIAG), Sektion "Gesteinsphysik und Bohrlochgeophysik“, Hannover

II. Neue Technologien zur Erforschung der unsichtbaren Welt von aquatischen Lebensräumen

Dozenten: Prof. Dr. Kirsten Küsel, Dr. Martina Herrmann, Dr. Carl-Eric Wegner (FSU Jena)

\section{Exkursionen}

Exkursion I: Sanierung der industriellen Absetzanlagen der Wismut GmbH am Standort Seelingstädt Leitung: Dipl.-Geol. Ulf Barnekow Termin: Mi 23.03.2022, 10.00 - ca. 18.00 Uhr

Exkursion II: Hainich-Exploratorium (Hainich Critical Zone Exploratory)

Leitung: Lehrstuhl Hydrogeologie der FSU Jena \& Nationalpark Hainich

Termin: Sa. $\quad 26.03 .2022, \quad 13.00$ 18.00 Uhr
Exkursion III: Salinares Tiefenwasser, Solegewinnung und Subrosion in Bad Frankenhausen und Umgebung

Leitung: Dr. Jörn Geletneky, Annett Peters (TLUBN)

Termin: Sa. 26.03.2022, 13.00 - ca. 18.00 Uhr

Exkursion IV: Wald - Wasser Wandel: Bedeutung und Vulnerabilität von Wald-/Forst-Grundwassereinzugsgebieten

Leitung: Lehrstuhl Hydrogeologie der FSU Jena \& Thüringen Forst

Termin: Sa. 26.03.2022, 13.00 - ca. $18.00 \mathrm{Uhr}$

Exkursion V: Tiefbrunnen und Wasserwerk für die Trinkwasserversorgung Jena

Leitung: Dipl. Ing. Christoph Wittich Termin: Sa. 26.03.2022, 13.00 - ca. 18.00 Uhr

\section{Forum Junge Hydrogeologen}

Studierenden und Promovierenden wird die Möglichkeit gegeben, in einer gesonderten Veranstaltung interessante Studienprojekte, Master- bzw. Doktorarbeiten vorzustellen. Wir wenden uns mit der Bitte an alle Hochschullehrer, ihre Studierenden und Promovierenden anzuregen mit Poster- bzw. Vortragsbeiträgen zum Gelingen dieser Veranstaltung beizutragen.

Bei Anmeldung eines Vortrags oder Posters im Forum Junger Hydrogeologen ist die Tagungsteilnahme für FH-DGGV-Mitglieder kostenfrei. Der beste Vortrag im Forum Junger Hydrogeologen wird mit einer Teilnahme an einer FH-DGGV-Fortbildungsveranstaltung im Jahr 2022/23 honoriert.

\section{Kontaktadressen}

Geschäftsstelle der FH-DGGV

Dr. Ruth Kaufmann-Knoke

Mühlweg 2

67434 Neustadt/Weinstr.

Telefon: +496321 484-784
Telefax: +49 6321 484-783

E-Mail: geschaeftsstelle@fh-dggv.de

Friedrich-Schiller-Universität Jena

Regina Piechnick

Burgweg 11

07749 Jena

Telefon +493641948621

E-Mail: regina.piechnick@uni-jena.de

Aktuelle Information wegen COVID-19

Die 28. Tagung der FH-DGGV ist als Präsenzveranstaltung in Jena geplant. Sollte sich im Vorfeld zeigen, dass die Entwicklung der COVID-19-Pandemie dies nicht zulässt, so wird die Veranstaltung voraussichtlich online stattfinden. Bitte verfolgen Sie deshalb die entsprechenden aktuellen Ankündigungen der Veranstalter über die Tagungs-Homepage und per E-Mail. Wir bitten um Verständnis, falls kurzfristige Veränderungen erforderlich sein werden.

Weitere Informationen, Anmeldung und Beitragseinreichung unter: www. fh-dggv.de/jena-2022

Das Organisationsteam der FH-DGGV-Tagung 2021

\section{Fortbildungsveranstaltung der FH-DGGV: Durchführung und Auswertung von Pumpversuchen, 25.-26.02.2022 und 07.- 08.10.2022 in Bad Soden- Salmünster}

Den klassischen Auswerteverfahren für Pumpversuche liegen vereinfachende Annahmen eines idealisierten Brunnens und eines homogenen, isotropen, unendlich ausgedehnten Grundwasserleiters zugrunde. In der Praxis enthalten Datensätze von Pumpversuchen jedoch auch Einflüsse des Brunnens bzw. des Bohrlochs, von speziellen Aquiferbedingungen wie leaky oder Porendränung oder der räumlichen $\mathrm{Pa}$ - 
rametervariabilität sowie Begrenzungen des Grundwasserleiters.

Mit den „Diagnostischen Plots“ steht eine Analysemethode zur Verfügung, die aufgrund ihrer größeren Sensitivität die Identifizierung verschiedener Fließphasen und damit eine sicherere Bestimmung des passenden Aquifermodells und der zugehörigen hydraulischen Parameter ermöglicht.

Der zweitägige Kurs bietet eine intensive Einführung in die Pumpversuchsauswertung mit „Diagnostischen Plots“. Im Kurs werden hierfür einfache Excel-basierte Tabellenkalkulationen und Diagramme verwendet. Übungen am PC vertiefen das Verständnis für die Dateninterpretation insbesondere für die Ansprache von Aquifertypen, Rändern, Schichtkopplungen, Heterogenitäten und regionalen Trends.

Der technische Teil des Kurses befasst sich mit der praktischen Vorbereitung und Durchführung von Pumpversuchen mit dem Ziel einer hohen Datenqualität und eines effizienten Arbeitsablaufs. Angesprochen und in Übungen vertieft werden die Versuchsplanung mit einer adäquaten Auslegung des Messprogramms, die Versuchstechnik sowie die Kostenkalkulation.

Der Kurs setzt keine Kenntnisse in der Pumpversuchsauswertung voraus, Grundkenntnisse in der Hydrogeologie und im Umgang mit Excel werden jedoch vorausgesetzt. Durch die behandelten Auswertemethoden bietet sich der Kurs auch für eine Auffrischung bzw. Vertiefung der bisherigen Erfahrung an. Für die erfolgreiche Kursteilnahme wird ein Zertifikat ausgestellt.

\section{Referenten}

Prof. Dr. Uwe Hekel ist Leiter des Fachbereichs Grundwasser bei der HPC AG in Rottenburg sowie Lehrbeauftragter am Geowissenschaftlichen Institut der Universität Tübingen.

Dr. Carsten Leven, Fachbereich Geowissenschaften an der Universität Tübingen, Arbeitsgruppe „Hydrogeologie“, unterrichtet u. a. Kurse in ange- wandter Hydrogeologie und Methoden der angewandten Geowissenschaften

\section{Informationen}

Anmeldeschluss ist der 24.01.2022 bzw. der 05.09.2022.

Der Kurs ist auf $\mathbf{1 6}$ Personen begrenzt. Die Teilnahmegebühr beträgt $720 €$ (für Mitglieder der FH-DGGV $610 €)$. Studierende zahlen $470 €$ (stud. Mitglieder der FH-DGGV $400 €)$. Diese Gebühren beinhalten die Veranstaltungsunterlagen sowie die Übernachtung in einem Tagungshotel einschließlich Vollpension, beginnend am ersten Kurstag mit dem Mittagessen. Die Veranstaltung endet jeweils am Folgetag gegen 15.30 Uhr.

Detaillierte Informationen entnehmen Sie bitte dem Faltblatt zur Veranstaltung bzw. der Internet-Seite der FH-DGGV (www.fh-dggv.de).

\section{Kontaktadresse}

Anmeldungen über die Geschäftsstelle der FH-DGGV:

Frau Dr. Maike Rüsgen

Telefon: +49 072148070470

E-Mail: maike.ruesgen@fh-dggv.de

\section{Fortbildungsveranstaltung der FH-DGGV: Beschaffenheit des Grundwassers - Anorganische Inhaltsstoffe, Prozesse, Berechnungen, 03.-04.03.2022 in Bad Soden-Salmünster}

Die Beschaffenheit des Grundwassers wird durch eine Vielzahl an Prozessen und Wechselwirkungen bestimmt. Dazu zählen u.a. Eintrag, Lösungsund Fällungsprozesse, Sorption und Ionenaustausch, Redoxprozesse. In der Summe führen diese Prozesse zu einer Zusammensetzung des Grundwassers, die spezifisch ist für die Region, für die Geologie des Grundwasserleiters, für die Grundwasserhydraulik, für die Teufe und das Alter. Diese Veranstaltung geht auf die Bedeutung dieser Prozesse für die Zusammensetzung des Grundwassers ein. Zunächst wird die Herkunft der Inhaltsstoffe beleuchtet und anschließend werden die wesentlichen Prozesse und Wechselwirkungen im Wasser und mit dem umgebenden Gestein behandelt. Die begleitenden Übungen dienen der Vertiefung und werden anhand von praktischen Beispielen durchgeführt.

Die Zielgruppe für diese Veranstaltung sind Vertreter und Vertreterinnen von Behörden, Ingenieurbüros und Versorgern. Grundkenntnisse in Chemie sind sinnvoll. Die Veranstaltung ist auch zur Auffrischung vorhandener Kenntnisse geeignet.

Die Veranstaltung gliedert sich in folgende Einheiten:

- Anorganische Beschaffenheit des Grundwassers: Herkunft der Ionen, Einteilung, Grenzwerte, Darstellung

- Lösungs- und Fällungsprozesse: Massenwirkungsgesetz, starke und schwache Säuren, Gase im Wasser, Kalk-Kohlensäure-Gleichgewicht, Sättigungsindex, Puffersysteme

- Sorption und Ionenaustausch: Sorbate und Sorbenten, Ionenaustausch, Sorptions-Isothermen

- Redoxprozesse: Nernst'sche Gleichung, Redoxreaktionen im Grundwasser, Redoxzonierung

\section{Referent}

Prof. Dr. Traugott Scheytt hat den Lehrstuhl für Hydrogeologie und Hydrochemie an der TU Bergakademie Freiberg. Er hält Vorlesungen zur Hydraulik, zur Hydrochemie und zum Stofftransport, betreut Masterarbeiten und Dissertationen und ist durch Publikationen und Vorträge wissenschaftlich ausgewiesen. 


\section{Informationen}

Anmeldeschluss ist der 4. Februar 2022. Die Teilnehmerzahl ist auf 20 Personen begrenzt.

Die Teilnahmegebühr beträgt $560 €$ (Mitglieder der FH-DGGV $480 €$ ). Studierende zahlen $360 €$ (stud. Mitglieder der FH-DGGV $310 €$ ). Diese Gebühren beinhalten die Kursgebühr, die Veranstaltungsunterlagen sowie die Übernachtung einschließlich Vollpension in einem Tagungshotel in Bad Soden-Salmünster.

Detaillierte Informationen entnehmen Sie bitte dem Flyer zur Veranstaltung bzw. der Internet-Seite der FHDGGV (www.fh-dggv.de).

\section{Kontaktadresse}

Anmeldungen über die Geschäftsstelle der FH-DGGV:

Frau Dr. Maike Rüsgen

Telefon: +49 72148070470

E-Mail: maike.ruesgen@fh-dggv.de

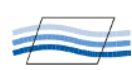

\section{Fortbildungsveranstaltung der FH-DGGV: Hydrogeochemische Systeme quantifizieren mit PHREEQC, 15.-17.03.2022 in Hildesheim}

Dieser Kurs befasst sich mit anwendungsnahen Simulationen zum Verständnis und zur Quantifizierung hydrogeochemischer Systeme. Die Tatsache, dass die Betrachtung und Bewertung der Umwelt innerhalb eines regulatorischen Rahmens durchgeführt werden, ist für das Verständnis der Rolle der geochemischen Modellierung und der Geochemie im Allgemeinen von wesentlicher Bedeutung. An dieser Stelle spielt gerade auch die Qualitätssicherung eine bedeutende Rolle. Um die beste Wissenschaft und die genausten Modelle in die Hände verantwortungsbewusster Anwender zu legen, wird die derzeitige Praxis basierend auf dem Stand der Technik der geochemischen Modellierung vermittelt. In dem Rahmen werden die Nützlichkeit und Einschränkungen der Modellkonzepte diskutiert und der ganzheitliche Blick auf geochemische Modelle geschult. Es ist das Ziel dieses Kurses, die akademische Forschung mit der Umweltpraxis zu verbinden und so praktizierenden Geochemikern und Geochemikerinnen, Hydrogeologen und Hydrogeologinnen, Ingenieuren und Ingenieurinnen sowie Aufsichtsbehörden einen Leitfaden zur geochemischen Modellierung zu bieten.

Die Teilnehmenden des Kurses erlernen die Nutzung des anwenderfreundlichen Computerprogramms PHREEQC. Dabei liegt der Fokus zunächst auf dem geochemischen Hintergrundwissen und der Rolle geochemischer Modellierung in der Praxis. Bei fast allen feldbezogenen Umweltanalysen besteht der Bedarf an Kenntnissen und/oder Vorhersagen der Konzentrationen gelöster Stoffe in Raum und Zeit mit Bezug zur Ressource Grundwasser oder in diesem Zusammenhang auftretenden Kontaminationsproblemen. Neben den Modellierungskonzepten, den thermodynamischen und kinetischen Grundlagen, werden Speziations-, Reaktionspfad und auch gekoppelte reaktive Transportmodelle im Kurs behandelt.

\section{Referent}

Prof. Dr. Michael Kühn hat Chemie und Geologie studiert. Er wurde 1997 an der Universität Bremen in Geochemie promoviert und hat sich 2003 an der TU Hamburg-Harburg für das Fach Hydrologie habilitiert. Michael Kühn arbeitet am Deutschen GeoForschungsZentrum GFZ in Potsdam und leitet dort die Abteilung Fluidsystemmodellierung. Gleichzeitig ist er Professor für Hydrogeologie an der Universität Potsdam. Sein Forschungsschwerpunkt liegt auf der Untersuchung des Verhaltens von Wasser und anderen Fluiden in den Sedimenten und Gesteinen des geologischen Untergrunds. Er quantifiziert insbesondere geochemische Fluid-Gesteins-Wechselwirkungen mit Simulationen gekoppelter Prozesse. Ziel ist die quantitative Bewertung von Fluidsystemen im Rahmen der Erkundung und der Nutzung von Georessourcen im unterirdischen Raum, auch unter Berücksichtigung ökologischer und ökonomischer Aspekte. Dabei stellt die Modellierung mit der Software PHREEQC ein wesentliches Hilfsmittel dar zum Verständnis aquatischer Systeme.

\section{Informationen}

Anmeldeschluss ist der 14. Februar 2022. Der Kurs ist auf 20 Personen begrenzt.

Die Teilnahmegebühr beträgt $1050 €$ (Mitglieder der FH-DGGV $930 €$ ). Studierende zahlen $710 €$ (stud. Mitglieder der FH-DGGV $600 €$ ). Diese Gebühren beinhalten die Kursgebühr, Veranstaltungsunterlagen sowie die Übernachtungen in einem Tagungshotel einschließlich Vollpension in Hildesheim. Die Veranstaltung beginnt am 15.03.2022 um 9.00 Uhr und endet am 17.03.2022 gegen 17.30 Uhr.

Detaillierte Informationen entnehmen Sie bitte dem Flyer zur Veranstaltung bzw. der Internet-Seite der FHDGGV (www.fh-dggv.de).

\section{Kontaktadresse}

Anmeldungen über die Geschäftsstelle der FH-DGGV:

Frau Dr. Maike Rüsgen

Telefon: +49 72148070470

E-Mail: maike.ruesgen@fh-dggv.de 


\section{Fortbildungsveranstaltung der FH-DGGV: Wasserhaushalts- untersuchungen - Grundlagen, Berechnungsmodelle, Anwendungsbeispiele, 07.-08.04.2022 in Bad Soden- Salmünster}

Die quantitative Beschreibung der Prozesse des Bodenwasserhaushalts auf der lokalen Maßstabsebene und die Modellierung ihres Zusammenwirkens in der Zeit ist Voraussetzung für die Beantwortung vieler hydrologischer und hydrogeologischer Fragestellungen. Der Kurs vermittelt wesentliche theoretische Grundlagen zu bodenhydrologischen Prozessen und Modellen. Darüber hinaus werden die Wasserhaushaltsmodelle BOWAHALD und HELP-D vorgestellt und ihre Anwendung für praktische Fragestellungen am eigenen Notebook eingeübt, die Ergebnisse diskutiert sowie Stärken und Schwächen der Modelle herausgestellt.

Die Zielgruppe des Kurses sind Mitarbeiterinnen und Mitarbeiter von Ingenieurbüros und Behörden, aber auch aus Universitäten. Grundkenntnisse in Hydrologie und/oder Bodenkunde sind sinnvoll, allerdings will diese Fortbildungsveranstaltung insbesondere die Grundlagen vermitteln. Sie ist deshalb auch zur Auffrischung vorhandener Kenntnisse geeignet. Die Schwerpunkte können in der Veranstaltung je nach Kenntnissen und Interessen der Teilnehmenden gesetzt werden.

Die Veranstaltung gliedert sich in folgende Einheiten:

- Notwendigkeit und Anwendungsbeispiele des Einsatzes von Bodenwasserhaushaltsmodellen auf der lokalen Maßstabsebene

- Grundlagen bodenhydrologischer Prozesse

- Einführung in bodenhydrologische Modelle und ihre Anwendung

- Was serhaushalts modelle BOWAHALD und HELP-D
- Übungen am eigenen Notebook mit den Modellen BOWAHALD und HELP-D

- Fazit: Möglichkeiten und Grenzen des Einsatzes von Wasserhaushaltsmodellen

\section{Referenten}

Dr. habil. Volkmar Dunger ist Dozent an der TU Bergakademie Freiberg, Professur für Hydrogeologie und Hydrochemie. Er hält Vorlesungen zur Hydrometrie, zum Wasserhaushalt und zur Hydropedologie, betreut studentische Qualifizierungsarbeiten und Promotionen, ist auf den Gebieten des Wasserhaushalts- und der Niederschlag-Abfluss-Modellierung aktiv und Entwickler des Wasserhaushaltsmodells BOWAM/BOWAHALD.

Dr. Klaus Berger ist Wissenschaftler im Institut für Bodenkunde der Universität Hamburg. Herr Berger hält Vorlesungen u. a. zur Bodenhydrologie und ist Autor der deutschen Weiterentwicklung des „Hydrologic Evaluation of Landfill Performance“ (HELP) Modells sowie Autor bzw. Coautor einer Vielzahl von Publikationen zum Wasserhaushalt insbesondere von Oberflächenabdichtungssystemen für Deponien und Altlasten.

\section{Informationen}

Anmeldeschluss ist der 7. März 2022. Die Teilnehmerzahl ist auf 20 Personen begrenzt.

Die Teilnahmegebühr beträgt $670 €$ (Mitglieder der FH-DGGV $580 €$ ). Studierende zahlen $440 €$ (stud. Mitglieder der FH-DGGV $370 €$ ). Diese Gebühren beinhalten die Kursgebühr, Veranstaltungsunterlagen sowie die Übernachtung in einem Tagungshotel einschließlich Vollpension. Die Veranstaltung endet am 08.04.2022 gegen 15.00 Uhr.

Detaillierte Informationen entnehmen Sie bitte dem Flyer zur Veranstaltung bzw. der Internet-Seite der FHDGGV (www.fh-dggv.de).
Die Nutzung der Programme im Kurs auf Rechnern der Teilnehmenden ist uneingeschränkt ohne Zusatzkosten möglich, die Kursteilnehmenden können beide Programme bis 14 Tage nach Kursende für die Bearbeitung der Kursaufgaben weiter nutzen.

Das Modell BOWAM/ BOWAHALD kostet als Vollversion 351,05€ incl. MwSt., die Kursteilnehmenden erhalten innerhalb von 14 Tagen nach Kursende einen Nachlass von $50 \%$.

Eine Lizenz von HELP-D kostet 233,95€ incl. MwSt., die Kursteilnehmenden erhalten innerhalb von $14 \mathrm{Ta}-$ gen nach Kursende einen Nachlass in der Form „,zwei Lizenzen zum Preis von einer".

\section{Kontaktadresse}

Anmeldungen über die Geschäftsstelle der FH-DGGV:

Frau Dr. Maike Rüsgen

Telefon: +49 72148070470

E-Mail: maike.ruesgen@fh-dggv.de

\section{Fortbildungsveranstaltung der FH-DGGV: Angewandte Grundwassermodellierung I - Einführung in die Strömungs- und Transportmodellierung mit praktischen Anwendungen am PC, 20.-23.04.2022 in Bad Soden-Salmünster}

Die numerische Grundwassermodellierung ist heute ein Standardwerkzeug zur Analyse und Prognose von Grundwasserströmung und Stofftransport. Die Anwendung reicht von lokalen Fragestellungen bezüglich der Sanierung von Grundwasserkontaminationen bis hin zur regionalen Grundwasserbewirtschaftung. Deshalb gibt es einen wachsenden Bedarf an Expertise in Hydrogeologie oder Bau- und Umweltingenieurwesen mit Kenntnissen in der Grundwassermodellierung. Mit 
den modernen leicht zu bedienenden Modellierungssystemen hat sich der notwendige Erfahrungshintergrund von der Programmierung und der Programmbedienung hin zu einer adäquaten, problemorientierten Umsetzung der Natur in ein Grundwassermodell verschoben.

Die FH-DGGV bietet mit drei aufeinander aufbauenden Kursen einen vertieften Zugang zur Modellierung von Grundwasserströmung und Stofftransport an.

Der 4-tägige Einführungskurs zur Grundwassermodellierung findet im April 2022 in Bad Soden-Salmünster statt. Dieser Intensivkurs bietet einen Zugang zur Strömungs- und Transportmodellierung, der speziell auf die Erstellung des hydrogeologischen Modells und den Aufbau des numerischen Modells zugeschnitten ist. Grundlagen werden erklärt, ohne im mathematischen Detail stecken zu bleiben. Übungsbeispiele reichen von typischen Sanierungsanwendungen bis zum regionalen Grundwassermanagement. Diese Übungen am PC bauen Hemmschwellen ab, vertiefen das Verständnis der Haupteinflussfaktoren auf Strömung und Transport und bieten praktische Erfahrung mit dem Aufbau und der Bewertung von Modellen.

Angesprochen sind Fachleute aus Hydrogeologie, Wasserwirtschaft, Umweltschutz und Grundbau, die z.B. in Ingenieurbüros, Behörden für Umweltschutz, Wasserwirtschaft und Geologie sowie Wasserversorgungsunternehmen tätig sind. Für die erfolgreiche Teilnahme wird ein Zertifikat ausgestellt.

In den Fortgeschrittenenkursen werden im zweiten Kurs die Themenbereiche Modellierung komplexer, dynamischer Systeme, Strömung und Stofftransport in Kluftsystemen (Doppelporositätsmodelle), numerische Stabilitätsanforderungen für den Stofftransport sowie chemische Reaktionen behandelt.
Dem Thema Kalibration und Parameteroptimierung ist ein eigener dritter Kurs gewidmet. Die Inhalte der Vorgängerkurse werden hier vorausgesetzt. Schwerpunkte sind der Umgang mit Nichteindeutigkeiten bei der Überlagerung verschiedener Fehlerquellen und die Bewertung der Prognosegenauigkeit von stationärer/transienter Strömung und Stofftransport. Eine Einführung in die automatisierte Parameteroptimierung erweitert die Optimierungsmöglichkeiten über den von der Modellierungs-Software vorgegebenen Parameterrahmen hinaus.

Details und Termine der Aufbaukurse entnehmen Sie bitte der Internetseite der FH-DGGV.

\section{Referierende}

Dr. Johannes Riegger, Institut für Wasser- und Umweltsystemmodellierung der Universität Stuttgart, Lehrstuhl „Hydrologie und Geohydrologie“, gibt Kurse in Geohydraulik, Grundwassermodellierung, hydrologische Modellierung, Geostatistik und GIS.

Dr. Anneli Guthke, Exzellenzcluster für ,Daten-integrierte Simulationswissenschaft" der Universität Stuttgart, unterrichtet in Grundwassermodellierung und Statistik.

\section{Informationen}

Anmeldeschluss ist der 21. März 2022.

Die Teilnahmegebühr beträgt $1420 €$ (Mitglieder der FH-DGGV $1200 €$ ). Studierende zahlen $920 €$ (stud. Mitglieder der FH-DGGV $780 €$ ). Diese Gebühren beinhalten die Kursgebühr, Veranstaltungsunterlagen sowie die Übernachtungen in einem Tagungshotel einschließlich Vollpension in Bad Soden-Salmünster. Eine Anreise am Vorabend kann mit gebucht werden.

Zum Kurs ist unbedingt ein eigener Laptop mitzubringen. Detaillierte Informationen entnehmen Sie bitte dem Flyer zur Veranstaltung bzw. der
Ein Termin, den Sie sich merken sollten:

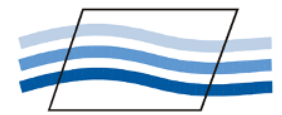

FH-DGGV-Fortbildung

Durchführung und Auswertung von Pumpversuchen

Bad Soden-Salmünster

25.-26.02.2022

Internet-Seite der FH-DGGV (www. fh-dggv.de).

\section{Kontaktadresse}

Anmeldungen über die Geschäftsstelle der FH-DGGV:

Frau Dr. Maike Rüsgen

Telefon: +49 72148070470

E-Mail: maike.ruesgen@fh-dggv.de

\section{Personalia}

\section{Dresdner Grundwasser- forschungspreis 2021 verliehen}

Zum Auftakt des Fachkolloquiums der Dresdner Grundwassertage 2021 hat die Stiftung zur Förderung der Dresdner „Wissenschaftlichen Schule Zunker-Busch-Luckner" (ZBL) den Dresdner Grundwasserforschungspreis 2021 vergeben. Die Preisverleihung fand im Rahmen der Veranstaltung, die vom Sächsischen Staatsminister für Energie, Klimaschutz, Umwelt und Landwirtschaft Wolfram Günter eröffnet wurde, am 8. Juni in Dresden statt.

Die Stiftung zeichnet in diesem Jahr zwei Preisträger mit dem Dresdner Grundwasserforschungspreis aus: Dr.-Ing. Johannes John und Dr.-Ing. Jonas Jurisch überzeugten die Jury unter Vorsitz von Prof. Dr. Georg Teutsch 
und den Beisitzern Prof. Dr. Rudolf Liedl und Prof. Dr. Frieder Häfner. Für den Preis, den die Stiftung für Promotionen auf den Gebieten Modellbildung und Simulation, der experimentellen Grundlagen- und Anwendungsforschung in Labor und Feld sowie der Beispiellösung pilothafter Praxisprobleme durch innovative Forschungsapplikation ausschreibt, wurden sechs gut begründete Anträge mit exzellenten Arbeiten eingereicht.

Die Dissertation von Dr.-Ing. Johannes John zum Thema „Konzeptionelle Modelle zur Beschreibung der Rheologie granularer Medien am Beispiel Lausitzer Fließsande" wurde unter der Betreuung von Prof. Dr. Rudolf Liedl erarbeitet und von der Fakultät Umweltwissenschaften der TU Dresden genehmigt. Entsprechend erfolgte die Genehmigung der Dissertation von Dr.-Ing. Jonas Jurisch zum Thema „Verfestigungsverhalten wassergesättigter und verflüssigter Granulate " unter der Betreuung von Prof. Dr. Boris Lehmann vom Fachbereich Bau- und Umweltingenieurwissenschaften der TU Darmstadt. Beide Dissertationen wurden von den Universitäten mit „magna cum laude“ bewertet.

Die von der Stiftung ZBL eingesetzte Jury hob in ihrer Begründung der Preisvergabe das hohe theoretische Niveau und die wissenschaftliche Akkuratesse der Dissertationsschriften bei der integralen strömungs- und bodenmechanischen Prozessanalyse besonders hervor. So formulierte Prof.

Ein Termin, den Sie sich merken sollten:

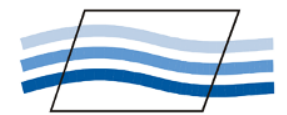

FH-DGGV-Fortbildung

Beschaffenheit des Grundwassers

Bad Soden-Salmünster

03. -04.03 .2022$
Dr. Frieder Häfner von der TU Bergakademie Freiberg in seinem Teilgutachten als Jury-Mitglied: „Nach meiner Kenntnis stellen die Dissertationen von Jurisch und John die ersten ihrer Art dar, die die bodenmechanische und die geoströmungs-mechanische Betrachtungsweisen (Mechanik von Granulaten, Mehrphasenströmung Wasser-Bodenluft) zusammenführen. (Der ,Vater' der Setzungsfließ-Bekämpfungstechnologien, der Bodenmechaniker und langjährige Geotechnik-Chef an der Bergakademie, Prof. Wolfang Förster, hat mir gegenüber schon vor langer Zeit bedauert, dass er nicht selbst diese Kopplung versucht hat.) Beide Aufgabenstellungen haben meines Erachtens einen hohen wissenschaftlichen Neuheitswert, der sie darin von den anderen [...] eingereichten Dissertationen unterscheidet."

Der Fachöffentlichkeit wurden die beiden Dissertationsschriften mit den Proceedings des DGFZ e.V. (ISSN 1430-0176, Hefte 54 und 55) zugänglich gemacht.

Die Stiftung zur Förderung der Dresdner „Wissenschaftlichen Schule Zunker-Busch-Luckner“ (ZBL) wurde 1993 durch Prof. Dr.-Ing. habil. Ludwig Luckner und Prof. Dr.-Ing. Dr.-Ing. habil. Dipl. Gwl. Karl Franz Busch zur Förderung von Forschung und Bildung zur Bewahrung des Umweltgutes Grundwasser errichtet. Sie ist die erstgegründete Umweltstiftung in den neuen Bundesländern. Die Stiftung fördert Wissenschaft sowie Aus- bzw. Weiterbildung auf dem Gebiet der Grundwasserforschung, um das unter dem besonderen Schutz der Allgemeinheit stehende Umweltgut Grundwasser zu bewahren. Hierfür wird alle zwei Jahre der Dresdner Grundwasserforschungspreis anlässlich der Dresdner Grundwasserforschungstage vergeben. Darüber hinaus finanziert die Stiftung kurze Gastaufenthalte von Wissenschaftlern am Dresdner Grundwasserforschungsinstitut, die sich dort speziell weiterbilden oder zum Beispiel ihre
Promotion konzipieren oder beenden können.

H. Sonntag, Dresden

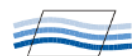

\section{Berichte und Informationen}

\section{Bericht vom 30. Doktorandentreffen der Hydrogeologie im Juli 2021}

Das 30. Doktorandentreffen der Hydrogeologie fand vom 14.07. bis zum 15.07.2021 aufgrund der Einschränkungen durch die COVID-19-Pandemie im Unterschied zu den vorherigen Jahren als Onlineveranstaltung statt. Gastgeber waren in diesem Jahr die Doktoranden und Doktorandinnen der Arbeitsgruppen der Angewandten Geologie der Christian-Albrechts-Universität (CAU) zu Kiel.

Der erste Tag des Doktorandentreffens startete mit einer Key Note von Prof. Dr. Andreas Dahmke, Leiter der Arbeitsgruppe für aquatische Geochemie und Hydrogeologie am Institut für Geowissenschaften in Kiel. Prof. Dr. Andreas Dahmke legte insbesondere einen Fokus auf zukünftige Arbeitsfelder von Hydrogeologen, insbesondere in Bezug auf die Herausforderungen des globalen Klimawandels sowie die Umstellung der Energieversorgung auf erneuerbare Energien. Vor allem die Nutzung des Untergrundes als saisonaler Wärmespeicher war Thema seines Vortrages.

Im Anschluss folgte ein Vortrag des Gastredners Mathias Foot zu der öffentlichkeitswirksamen Darstellung wissenschaftlicher Daten. Im Anschluss an den Vortrag diskutierte er mit den teilnehmenden Doktoranden und Doktorandinnen über die Möglichkeiten, ihre Forschungsergebnisse verständlich für Interessierte außerhalb des eigenen Fachbereichs aufzubereiten. 
Im weiteren Verlauf folgten neun Beiträge der teilnehmenden Doktoranden und Doktorandinnen von der TU Freiberg, dem Karlsruher Institut für Technologie, der FU Berlin, der FAU Erlangen, dem Geozentrum Hannover, dem GFZ Potsdam und der CAU Kiel in Form von Vorträgen zu den Themen Wasser bzw. Grundwassermanagement und -monitoring sowie zu der Hydrochemie von Untergrundwärmespeichern. Im Anschluss an die Vorträge folgten spannende Diskussionen $\mathrm{zu}$ roboterunterstützten Wasserprobennahmen in Seen, (Grund-)Wassermanagement in Vietnam und Indien, gelöstem Sauerstoff in aquatischen Ökosystemen, Methodiken zur Bestimmung des Grundwasseralters, dem Transport von Mikroplastik in Böden sowie zu hydrochemischen Auswirkungen der Nutzung des Untergrundes als Wärmespeicher.

Der zweite Tag stand ebenfalls im Zeichen der erneuerbaren Energien und startete mit einem Gastvortrag von Dr. Klaus Wortmann (EKSH) zu Szenarien-Modellen der Energiewende sowie Energielandschaften in Schleswig-Holstein. Dabei wurden Zukunftsvisionen zur Klimaneutralität in urbanen Räumen aber auch länderübergreifend diskutiert und die Frage aufgegriffen, welche Aufgaben künftig die Hydrogeologie zur Bewältigung der Energiewende beschäftigen werden. Weiterhin wurde in Verbindung zum Gastvortrag des 1. Tages das Thema Wissenschaftskommunikation aufgegriffen und eine Diskussion darüber geführt, wie man Ergebnisse aus Forschung und Wissenschaft, wie beispielsweise Zukunftsszenarien zur Klimaneutralität, an die weite Bevölkerung bringen kann und wie die Resonanz zu solchen Projekten ist.

Im weiteren Tagesverlauf folgten Beiträge der teilnehmenden Doktoranden und Doktorandinnen in Form von Vorträgen zum Schwerpunkt numerische Modellierung, sowie analytische Methoden im Feld von insgesamt sieben Teilnehmern von der CAU Kiel,

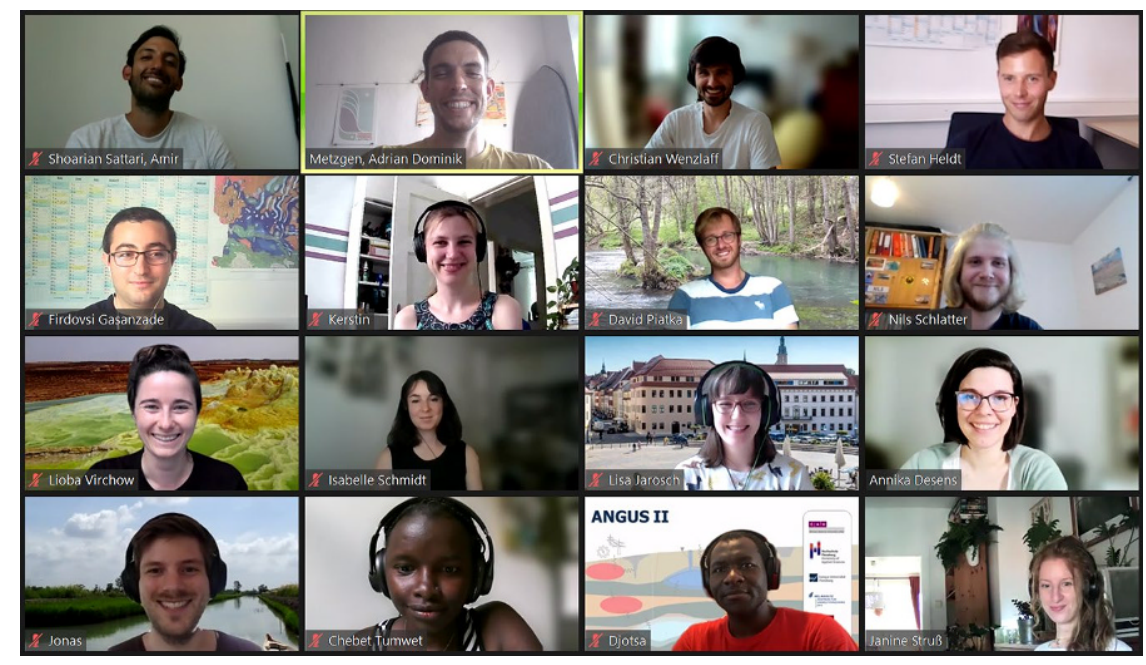

Abb. 2 Screenshot 30. Doktorandentreffen Kiel

dem GFZ Potsdam und der RWTH Aachen. Insgesamt konnte an diesem Tag ein spannender wissenschaftlicher Diskurs zu Themen wie die Verwendung erneuerbarer Energien zur saisonalen Wärmespeicherung mithilfe von Aquifer- und Erdwärmesondenspeichern und zur Druckluftenergiespeicherung, die geomechanische Beschaffenheit geologischer Reservoir- und Barrierestrukturen sowie ein Einblick in die laserinduzierte Plasmaspektroskopie als Analysemethode im Feld abgehalten werden.

Das 30. Doktorandentreffen der Hydrogeologie (Abb. 2) stellte auch in diesem Jahr trotz pandemiebedingter Online-Veranstaltung wieder eine schöne und wertvolle Plattform für alle teilnehmenden Doktoranden und Doktorandinnen dar, sich wissenschaftlich über die eigenen Forschungsthemen hinaus auszutauschen und damit auch die Themengebiete und Arbeitsweisen anderer Arbeitsgruppen kennen zu lernen. Da es bei allen Teilnehmern und Teilnehmerinnen großen Anklang gefunden hat, wurde beschlossen das kommende 31. Doktorandentreffen 2022 unter dem Zusammenschluss des GFZ Potsdam und der TU Berlin in Berlin stattfinden zu lassen.

Das 30. Doktorandentreffen wurde freundlicherweise vom Kompetenzzentrum Geo-Energie, der Fachsekti- on Hydrogeologie in der DGGV (FHDGGV), der Gesellschaft für Energie und Klimaschutz Schleswig-Holstein (EKSH) sowie der International Association of Hydrogeologists (IAH) unterstützt. Wir danken herzlich den Gastrednern Mathias Foot und Dr. Klaus Wortmann für ihre großartigen Beiträge. Ein besonderer Dank gilt auch dem Key-Note Speaker Prof. Dr. Andreas Dahmke, der vor ca. 30 Jahren die Tradition der hydrogeologischen Doktorandentreffen ins Leben gerufen hat.

A. Metzgen, J. Struss \& K. Meier zu Beerentrup, Kiel

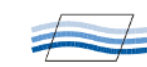

Ein Termin, den Sie sich merken sollten:

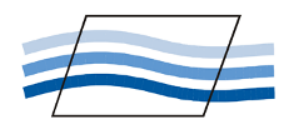

FH-DGGV-Fortbildung

Hydrogeochemische Systeme quantifizieren mit PHREEQC

Hildesheim

15.-17.03.2022 


\section{Forum}

\section{Fachsektion Hydrogeologie}

\section{In eigener Sache}

Liebe Kollegen und Kolleginnen, im Forum stellen wir Stellungnahmen zu aktuellen Themen für unsere Mitglieder zur Diskussion. Gerne nehmen wir Ihre Beiträge - beispielsweise als Leserbriefe - entgegen. Wir freuen uns auf eine intensive Diskussion! Ihre Beiträge senden Sie bitte an:

Patricia Schüll

E-Mail: p.schuell@gmx.de

Vielen Dank für Ihre Unterstützung!

Die Redaktion

\section{Es stellt sich vor}

\section{Interdisziplinäres DFG-Projekt Bad-Waldsee gestartet}

Mit einer Bohrung zur Gewinnung von Sedimentkernen aus dem See an der oberschwäbischen Stadt Bad Waldsee startete im Juni 2021 ein interdisziplinäres DFG-Projekt, an dem jeweils wissenschaftliche Mitarbeitende der Technischen Universität Braunschweig, der Technischen Universität Darmstadt und der Universität Tübingen sowie des Landesamts für Denkmalpflege Baden-Württemberg beteiligt sind.

Das Forschungsvorhaben zeichnet sich durch die enge Zusammenarbeit von Geowissenschaften, der Geschichtswissenschaft, der Archäologie sowie der Archäobotanik aus. Wirtschafts-, sozial- und umweltgeschichtliche Phänomene werden in diesem Projekt erstmals gemeinsam von Geistes- und Naturwissenschaften untersucht und interpretiert. Neben dem Sedimentarchiv werden als Archive auch Schriftquellen, Baumringe und archäologische Ausgrabungen erforscht und Ereignisse in den Archiven in Bezug zueinander gesetzt.

Bad Waldsee ermöglicht eine europaweit einzigartige Studie, da die Stadtentwicklung unmittelbar am See ablief und dessen Sedimente die Stadtentwicklung in Jahreslagen aufzeichnete. Dadurch können erstmals die Auswirkungen einer mittelalterlichen bis frühneuzeitlichen Stadtentwicklung (Zeitraum zwischen ca. 1200 und 1800 AD) auf Gewässer in hoher Auflösung dargestellt werden. Neben den anthropogenen Einflüssen sollen auch natürliche Witterungsereignisse und die klimatische Entwicklung verfolgt werden, um den Einfluss der natürlichen Rahmenbedingungen besser zu verstehen.

Die Geowissenschaftler und Geowissenschaftlerinnen der TU Darmstadt Kristin Hass und AOR Dr. Thomas Schiedek untersuchen unter der Leitung von Prof. Dr. Matthias Hinderer (Institut für Angewandte Geowissenschaften, Arbeitsgruppe Angewandte Sedimentgeologie) die Bohrkerne auf sedimentologische und geochemische Parameter, darunter auch organische Schadstoffe wie z.B. polyzyklische, aromatische Kohlenwasserstoffe (PAK). Diese wurden einerseits durch Waldbrände (anthropogen und natürlich), aber vor allem auch bei Stadtbränden oder dem winterlichen Hausbrand emittiert und durch natürliche Deposition in den See eingebracht und sedimentiert. Darüber hinaus werden Elemente untersucht, die als Emissionen von Handwerksbetrieben (z.B. Gerbereien) bekannt sind. Es wird daher erwartet, dass eine jahrgenaue Zuordnung der Gewässerverschmutzungen zu Ereignissen der mittelalterlichen Stadtentwicklung hergestellt werden kann.
T. Schiedek, K. Haas \& M. Hinderer, Darmstadt

\section{Schutzfunktion der Grundwasserüberdeckung - Methoden und Anwendbarkeit}

Der nachfolgende Text ist die Kurzfassung eines Berichts, der von der AG Hydrogeologie der Staatlichen Geologischen Dienste in Deutschland (SGD) bestehend aus den geologischen Landesämtern der Bundesländer und der Bundesanstalt für Geowissenschaften und Rohstoffe (BGR) erarbeitet wurde.

Der komplette Bericht und die Anlagen stehen unter: https://www. infogeo.de/Infogeo/DE/Downloads/ AG_hydrogeologie_AA-HY2_ abschlussbericht.html

\section{Ausgangssituation}

Grundwasser ist in Deutschland die wichtigste Ressource für die öffentliche Wasserversorgung. Der Klimawandel kann jedoch in einigen Regionen Deutschlands dazu führen, dass das Grundwasser aufgrund einer möglichen zurückgehenden Grundwasserneubildung und insbesondere aufgrund eines steigenden Bedarfs knapp wird. Dem Erhalt der Grundwasserqualität muss daher höchste Priorität eingeräumt werden. Der Schutz des Grundwassers vor schädlichen Verunreinigungen wird so z.B. durch die Ausweisung von Wasserschutzgebieten, die Beseitigung bzw. Sicherung von Altlasten und Altstandorten sowie die Implementierung zunehmend strengerer Regelwerke verbessert. Die Auswirkungen dieser Maßnahmen 
werden auf Länderebene im Rahmen großräumiger Grundwasserbeobachtungen laufend überwacht.

Vor diesem Hintergrund sind Arbeiten und Untersuchungen über die potenzielle Schutzfunktion der die Grundwasserleiter überdeckenden Schichten erforderlich, damit Gefährdungspotenziale auf Grund unerwünschter Stoffeinträge z.B. durch die landwirtschaftliche Düngung, aus Altlasten und Altstandorten, durch Bergbaufolgelandschaften, aber auch aus Punktquellen im Einzelfall abgeschätzt werden können. In Wassergewinnungsgebieten stellt sich immer wieder die Frage, ob geplante Eingriffe in den Untergrund wie z.B. durch Baumaßnahmen das zur Trinkwasserversorgung genutzte Grundwasservorkommen schädigen können. Die Schutzfunktion der Grundwasserüberdeckung ist vor diesem Hintergrund eine sehr wichtige Beurteilungsgröße.

Die AG Hydrogeologie der Staatlichen Geologische Dienste in Deutschland (SGD) hat sich daher in den letzten Jahren u.a. mit unterschiedlichen Methoden zur Ermittlung der Schutzfunktion der Grundwasserüberdeckung hinsichtlich Anwendbarkeit, verfügbarer Datengrundlagen und Fortentwicklungsbedarf beschäftigt. Die in Deutschland wahrscheinlich am häufigsten angewandte Methode ist das „Konzept zur Ermittlung der Schutzfunktion der Grundwasserüberdeckung“" von Hölting et al. (1995). Die Methode wurde Anfang der 1990er Jahre von der AG Hydrogeologie erarbeitet und wird im Folgenden als SGD-Methode bezeichnet. Sie ist auch als Hölting-Methode bekannt.

\section{SGD-Methode}

Bei der SGD-Methode handelt es sich um einen intrinsischen Ansatz, bei dem der Hauptfaktor der Betrachtung die mittlere Verweilzeit eines idealen Tracers darstellt, der im Boden und der ungesättigten Zone mit dem Niederschlagswasser in das Grundwasser verlagert wird. Je länger ein Schadstoff braucht, um ins Grundwasser zu gelangen, umso mehr wird er von Prozessen wie z.B. Verdünnung, Sorption, Dispersion oder Abbau beeinflusst.

Die SGD-Methode ermöglicht keine stoffspezifische Bewertung für z. B. das Nitrat-, Pflanzenschutzmittel- oder Schwermetallrückhaltevermögen. Das Verfahren berücksichtigt auch keine potenziellen Reinigungsvorgänge.

Als wesentliche Parameter gehen die nutzbare Feldkapazität, die Sickerwassermenge, die Gesteinsart, die Mächtigkeit der Grundwasserüberdeckung, schwebende Grundwasserstockwerke und die Druckverhältnisse ein. Über ein empirisches Punktesystem wird eine klassifizierte Gesamtschutzfunktion berechnet.

Die SGD-Methode ermöglicht mit relativ einfachen Mitteln Betrachtungen der Schutzfunktion. Trotz der vereinfachten Betrachtung der eigentlich komplexeren Verhältnisse, ist es ein robustes, in der Fläche und für unterschiedliche Grundwasserlandschaften gleichermaßen anwendbares Bewertungsverfahren (Diepolder, 1995). Die SGD-Methode ermöglicht damit grundsätzlich die Erstellung landeseinheitlicher Kartenwerke.

Durch die relativ einfach zu beschaffenden oder abzuleitenden Eingangsdaten ist das Verfahren auch bei geringer Datendichte und ohne aufwendige Ermittlung zusätzlicher, oft nicht routinemäßig ermittelbarer $\mathrm{Pa}$ rameter anwendbar. Das Punktesystem und die Verknüpfungsvorschrift ermöglichen Berechnungsverfahren z. B. für Einzelbohrungen, für größere Gebiete, aber auch etwa rasterbasierte Bearbeitungen mit Hilfe von geographischen Informationssystemen.

Die Skalierung der Berechnungsergebnisse erfolgt von geringen Punktzahlen (=geringe Schutzfunktion) bis zu hohen Punktzahlen (=hohe Schutzfunktion). Zielgröße der Schutzfunktionsbewertung ist die Verweildauer des Sickerwassers in der Grundwasserüberdeckung (ungesättigte Zone). Die ermittelten Schutzfunktionspunktwerte werden deshalb in mittlere Verweilzeiten übersetzt (Tab. 1).

Durch die Reduktion der Komplexität und die Beschränkung auf die vertikale Komponente der Schadstoffverlagerung sind vergleichsweise übersichtliche, zweidimensionale Kartendarstellungen möglich. Die Betrachtung und Kombination von vertikalen und lateralen Verlagerungsprozessen würde neben den oft hierzu fehlenden Eingangsdaten und den dafür aufwendigen Berechnungen $\mathrm{zu}$ komplexeren und deutlich schwieriger visualisierbaren Ergebnissen führen.

In den letzten beiden Dekaden wurde national und international eine Vielzahl von Methoden mit ähnlichen Zielsetzungen erarbeitet. Für viele Gebiete gibt es mittlerweile digital verfügbare Grundlagendaten mit einem höheren Grad an Flächendeckung und Qualität, als dies Anfang der 1990er Jahren der Fall war. Durch stark verbesserte Rechnerleistungen sowie den Entwicklungen im Bereich der geographischen Informationssysteme, 3D-Modellierungen oder numerischen Modellierungsverfahren sind heute andere Bearbeitungsmöglichkeiten gegeben.

Durch die Umsetzung der SGDMethode in einigen Flächenländern (u. a. Bayern, Hessen, Baden-Württemberg, Sachsen, Brandenburg) liegen langjährige praktische Erfahrungen vor. Bei der Anwendung zeigte sich, dass einzelne Faktoren der Me-

Ein Termin, den Sie sich merken sollten:

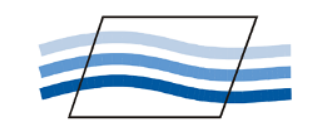

FH-DGGV-Fortbildung

Bohrlochgeophysik in der Praxis vom Messwert zum Ergebnis Jena

23.03.2022 
Tab. 1 Klassifizierung der Punktzahlen der Gesamtschutzfunktion und Interpretation der mittleren Verweildauer des Sickerwassers in der Grundwasserüberdeckung nach Hölting et al. (1995)

\begin{tabular}{llll}
\hline Klasse & $\begin{array}{l}\text { Punktzahl der Gesamtschutz- } \\
\text { funktion }\end{array}$ & Gesamtschutzfunktion verbal & $\begin{array}{l}\text { Größenordnung der mittleren Verweildauer des Sickerwassers in } \\
\text { der Grundwasserüberdeckung (bis zum Erreichen der Grund- } \\
\text { wasseroberfläche des bewerteten Grundwasserleiters) }\end{array}$ \\
\hline 0 & keine Daten & nicht berechnet & Oberflächengewässer/nicht definierte Bereiche \\
1 & $\leq 500$ & sehr gering & wenige Tage bis etwa 1 Jahr \\
2 & $>500-1000$ & gering & mehrere Monate bis ca. 3 Jahre \\
3 & $>1000-2000$ & mittel & 3-10 Jahre \\
4 & $>2000-4000$ & hoch & 10-25 Jahre \\
5 & $>4000$ & sehr hoch & $>25$ Jahre \\
\hline
\end{tabular}

thode in den Ländern teilweise unterschiedlich gehandhabt werden und insbesondere in Grundwasserleitern im Kristallin oder sedimentären Festgesteinen methodische Ergänzungen oder Abwandlungen erfolgten. Dies führte $\mathrm{zu}$ Weiterentwicklungen der SGD-Methode z.B. zu stoffspezifischen Bewertungsmöglichkeiten oder die Einbeziehung lateraler Komponenten sowie zur Übertragung auf andere Maßstabsbereiche.

\section{Umsetzung in den Bundesländern}

Auf Grund vielfältiger, oft konkurrierender Nutzungen des Untergrundes und der daraus resultierenden hohen Nutzungsdichte bilden flächenhafte Informationen zur Schutzfunktion der Grundwasserüberdeckung eine wichtige geowissenschaftliche Planungsgrundlage, so dass entsprechende Daten von allen SGD erarbeitet wurden. Allerdings kamen bei der Ausweisung der Schutzfunktion der Grundwasser-

\section{Ein Termin, den Sie sich} merken sollten:

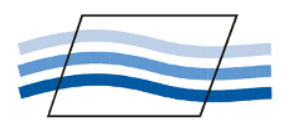

FH-DGGV-Fortbildung

Neue Technologien zur Erforschung der unsichtbaren Welt von aquatischen Lebensräumen Jena

23.03.2022 überdeckung in den Bundesländern unterschiedliche Verfahren zum Einsatz (s. Abb. 3), was u.a. zu Problemen bei bundeslandübergreifenden Fragestellungen führt.

Die Kartenmaßstäbe variieren zwischen 1:5000 und 1:500.000, wobei bei Maßstäben <1:100.000 erfahrungsgemäß die wachsende Unschärfe der Eingangsdaten (z. B. Grundwasserleiter-bezogener Flurabstand) das Ergebnis deutlich beeinflusst. Teilweise liegen Schutzfunktionskarten in den Ländern auch in unterschiedlichen Maßstäben vor. In acht Bundesländern stehen die Karten landesweit als digitale Datensätze zur Verfügung. In Baden-Württemberg, Berlin, Hessen, Niedersachsen, Thüringen und Sachsen sind Online-Zugriffe auf die Karten möglich.

Die Bearbeitungsstände datieren häufig vor 2007. In fünf Bundesländern laufen derzeit Arbeiten. Ein Bundesland plant diese. Brandenburg, Bayern, Bremen, Niedersachsen, Nordrhein-Westfalen und Sachsen erarbeiten aktuell Fachdatenbestände zur Schutzfunktion der Grundwasserüberdeckung, teilweise bereits auf der Grundlage von geologischen 3D-Modellen.

\section{Alternative Methoden}

Die Vulnerabilität bewertet die Verschmutzungsempfindlichkeit eines Grundwasserleiters gegenüber anthropogenen Einflüssen, während sich die Schutzfunktion auf die Grundwasserüberdeckung bezieht. Beide Ansätze beschreiben somit die Gefährdungssituation des vom Nutzer vorgegeben Bewertungshorizonts (z. B. des obersten Grundwasserleiters oder tieferer Zirkulationssysteme) auf unterschiedliche Weise. Tab. 2 zeigt beispielhaft eine Auswahl von Definitionen unterschiedlicher Autoren und Institutionen.

Insgesamt wurden mehr als $50 \mathrm{Me}$ thoden identifiziert. Auffällig ist, dass nur ein Bruchteil der aufgeführten Methoden in den letzten 10 Jahren veröffentlicht wurde. Daraus lässt sich ein gewisser Konsens bezüglich der Anwendbarkeit und Belastbarkeit der existierenden Methoden ableiten.

\section{Zusammenfassung und Ergebnisse}

Von Geologischen Diensten wurde recherchiert, welche Methoden angewendet werden oder zukünftig angewendet werden sollen und welche Eingangsdaten in die Berechnungen einfließen. Die Ergebnisse liegen tabellarisch zusammengefasst vor und geben einen guten Überblick über die mittlerweile entwickelten Modifikationen und Ergänzungen der ursprünglichen SGD-Methode.

Weiterhin wurden durch die BGR über 50 national und international publizierte Methoden zur Ermittlung der Schutzfunktion der Grundwasserüberdeckung bzw. der Vulnerabilität von Grundwasserleitern sowie die jeweils erforderlichen Eingangsdaten recherchiert. Dies erfolgte in Synergie zu Arbeiten im Rahmen des GeoERAVorhabens HOVER (Hydrogeological 
processes and geological settings over Europe controlling dissolved geogenic and anthropogenic elements in groundwater of relevance to human health and the status of dependent ecosystems). Als Ergebnis liegt eine umfangreiche Literatur- und Methodenübersicht vor.

Aus diesem Methodenpool wurden folgende, mit der SGD-Methode hinsichtlich Vorgehensweise und erforderlicher Eingangsdaten vergleichbaren und erfolgversprechenden Methoden ausgewählt und im Rahmen von drei Masterarbeiten (Liebhold 2018; Hengsberger 2018; Erber 2019) mit der SGD-Methode als Referenzsystem verglichen:

- DRASTIC-Methode (Aller et al. 1987)

- Hydrogeologische Karte 1:50.000 der DDR (Voigt 1987); Ermittlung der Grundwassergeschütztheit „HK50-Methode“

- LAWA-Methode (LAWA 2003)

- Methode nach Haertlé und Josopait (1980)

- DIN 19732 (Berechnung Verweilzeit des Sickerwassers in der Grundwasserüberdeckung)

- Methode des Umweltbundesamts (UBA 2002).

Ziel war der Gewinn von Erkenntnissen zur Übertragbarkeit der Ergebnisse in Modellgebieten in Mecklenburg-Vorpommern (Liebhold 2018), Hessen (Hengsberger 2018), Brandenburg (Erber 2019) und Niedersachsen. Schwerpunkte waren systematische Betrachtungen (z.B. intrinsische und spezifische Schutzfunktion; Vulnerabilität) sowie Methodenvergleiche
Abb. 3 Übersicht der in den Bundesländern eingesetzten Verfahren zur Ausweisung der Schutzfunktion der Grundwasserüberdeckung (verändert nach Erber, 2019)

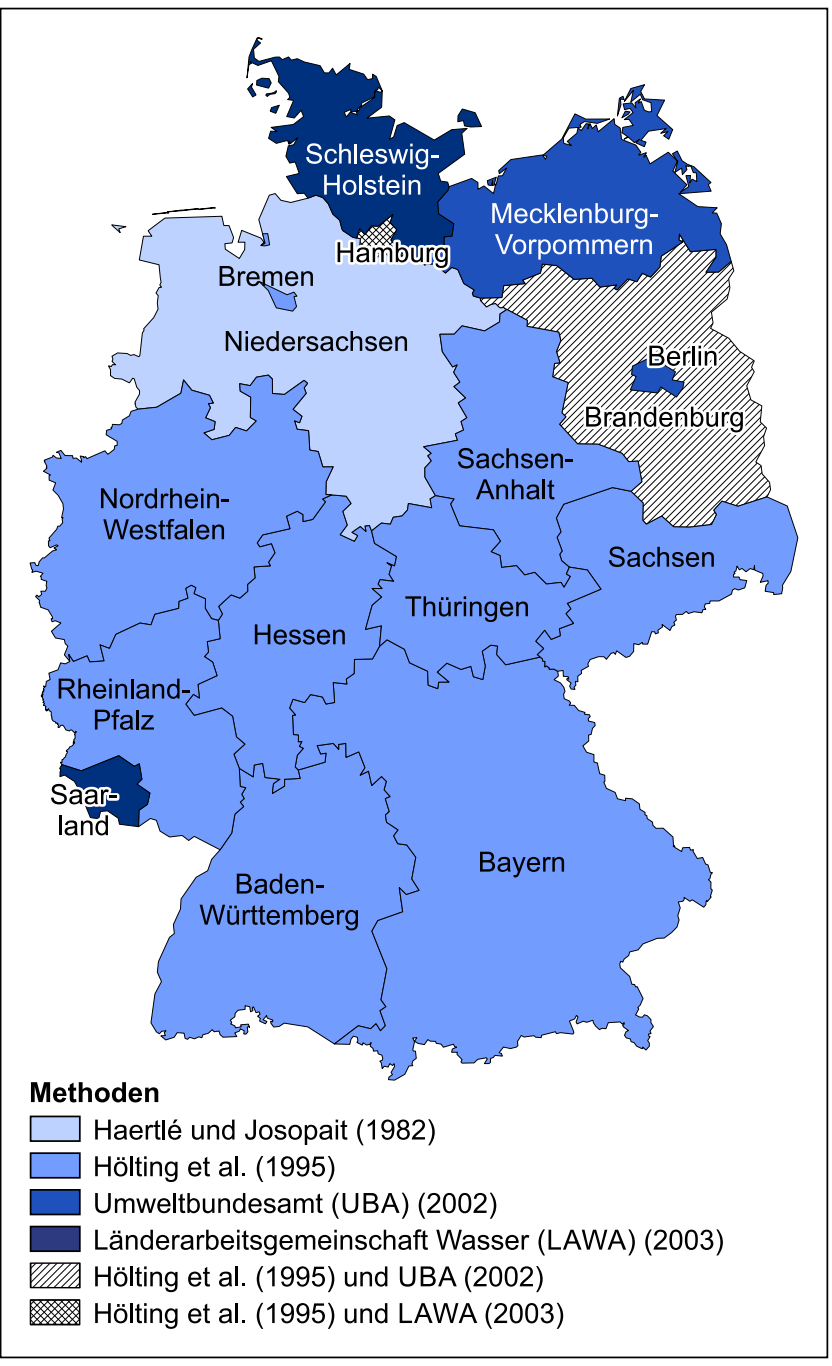

unter Berücksichtigung der Eingangsdaten, Anwendungserfahrung und auf Basis unterschiedlicher Ansätze von Reklassifizierungsmethoden.

Die SGD-Methode erweist sich insbesondere für Flächenstaaten als in der Praxis praktikabel umsetzbare und gut vergleichbare Methode zur Ermittlung der Schutzfunktion der Grundwasserüberdeckung.
Das Ergebnis des Methodenvergleichs zeigt, dass die Schutzfunktionsbewertung nach der SGD-Methode, DRASTIC und der HK50-Methode für Lockergesteinsbereiche in der kleinmaßstäblichen Betrachtung weitgehend vergleichbar ist. Für Festgesteinsbereiche stellt nur die SGD-Methode ein geeignetes Werkzeug für eine Bewertung dar. An-

Tab. 2 Auswahl von Definitionen der Verschmutzungsempfindlichkeit des Grundwassers unterschiedlicher Autoren und Institutionen

U.S. National Research Council „Groundwater vulnerability is not a measurable property, but a probability statement about future contamina(1993) tion that must be inferred by surrogate measures [...and] is best expressed as a probability to an event."

Zaporozec (2002)

Foster (1998)

Adam, Gläßer und Hölting (2000)
„Vulnerability is an intrinsic property of a groundwater system that depends on the sensitivity of that system to human and/or natural impact."

„Groundwater everywhere is vulnerable to surface-derived pollution and the only thing that differs geographi"cally is the time-scale for effects to become manifest."

„... Schutzfunktion der jeweils grundwasserüberdeckenden Schichten ..., die durch Art (Gesteinsausbildung, Sorptions- und Ionenaustauschkapazität, mikrobiologische Aktivität) und Mächtigkeit bestimmt wird ..." 
gesichts des prozentual großen Flächenanteils von Festgesteinen in der Bundesrepublik bestätigen die aktuellen Studien die damaligen Ergebnisse der Hölting-Arbeitsgruppe (1995), dass nur die SGD-Methode (Hölting et al. 1995) die Möglichkeit einer bundesweit methodisch einheitlichen Bewertung bietet.

Auf Grund der Robustheit der Methode können auch mittlerweile bestehende Modifikationen, Ergänzungen oder Abwandlungen meist problemlos integriert werden, ohne dass das Grundkonzept gefährdet wird. Hierbei handelt es sich vorwiegend um Ansätze, die über Regional- oder Korrekturfaktoren den Einfluss von strukturgeologischen Aspekten wie Störungen in Festgesteinsgebieten, von Verwitterungsbildungen, von Dolinen oder Versinkungsstellen sowie natürlichen oder durch Bergbau entstandenen unterirdischen Hohlräumen berücksichtigen.

Sehr hilfreich sind Ansätze und Methoden zur Ermittlung der Flurabstände (bzw. des Bezugshorizontes) im Festgestein oder für Gebiete mit geringen Grundwasservorkommen. In diesem Zusammenhang sind Klarstellungen hinsichtlich des bewerteten Grundwasserleiters bzw. betrachteten „Bewertungshorizonts“ erforderlich. Diese können dabei der oberste Grundwasserleiter unabhängig von der Nutzung oder das erste tatsächlich wasserwirtschaftlich relevante und genutzte Grundwasservorkommen sein. In einigen Ländern existieren digitale Ansätze und Algorithmen, die auch eine Berechnung der Schutzfunktion bis zu einem vom Benutzer gewählten Bewertungshorizont (beliebiger Grundwasserleiter) erlauben.

Hinsichtlich der Anwendbarkeit der SGD-Methode wird empfohlen, dem Nutzer ergänzende Hinweise zu Einschränkungen und Aussageschärfe der Methode sowie Empfehlungen zum Anwendungsbereich, dem „Use case“ und zur Skalierbarkeit/zum Maßstab zu geben. Die Güte des Berechnungs- ergebnisses (i.W. empirischer Punktwert der Schutzfunktion) ist in hohem Maße abhängig von der Qualität, der räumlichen Repräsentanz und der Auflösung der Ausgangsdaten.

Die SGD-Methode ist gut geeignet für mittlere und kleinere Maßstabsbereiche, d.h. 1:50.000 und kleiner. Typische Anwendungsfälle sind beispielsweise Regionalplanungen wie der Landschaftsrahmenplan in Baden-Württemberg oder der Regionalplan in Sachsen. Jedoch ist hierbei immer zu berücksichtigen, in welchem Maßstab Eingangsparameter eingehen. Dies kann dazu führen, dass je nach Datengrundlage in den Ländern unterschiedliche Maßstabsbereiche für belastbare Aussagen zur Schutzfunktion der Grundwasserüberdeckung vorgegeben werden.

Zur methodischen Harmonisierung ist die Fortschreibung und länderübergreifende Abstimmung der Lithopunktetabelle oder - bei pauschaleren Ansätzen - die Punktezuweisung ganzer hydrogeologischer Einheiten erforderlich, um unterschiedliche Bewertungen gleicher Formationen an administrativen Grenzen zu vermeiden.

\section{Literatur}

Adam, Ch., Gläßer, W., Hölting, B: Hydrogeologisches Wörterbuch; Enke Stuttgart - New York (2000)

Aller, L., Bennet, T., Lehr, J., Petty, R. Hackett, G.: DRASTIC: a standardized system for evaluating groundwater pollution potential using hydrogeological setting. - US-EPA Report 600/2-87/035. US Environmental Protection Agency, Oklahoma (1987)

DIN 19732: Bodenbeschaffenheit - Bestimmung des standörtlichen Verlagerungspotentials von nichtsorbierbaren Stoffen, 12 S. (2011)

Diepolder, G. W.: Schutzfunktion der Grundwasserüberdeckung: Grundlagen - Bewertung - Darstellung in Karten; GLA Fachberichte 13, 5-79; Bayerisches Geologisches Landesamt; München 1995 (1995)

Erber, A.: Vergleichende Analyse und Validierung flächenhafter Bewertungsmethoden der Grundwasservulnerabilität gegenüber Verschmutzung am Beispiel Nordost-Brandenburg. 192 S. Masterarbeit (2019, unveröffentl.)
Foster, S.S.D.: Fundamental concepts in aquifer vulnerability, pollution risk and protection strategy. In: van Duijvenbooden, W., van Wageningh, H.G. (Hrsg.): Vulnerability of soil and groundwater to pollutants. Proceed. of international conference, Noordwijk, The Netherlands, 69-86 (1987)

Haertlé, T., Josopait, V.: Methodik und Arbeitsweise zur Anfertigung von Karten über die natürlichen Grundwasserschutzbedingungen. In: Institut für Stadtbauwesen der TU Braunschweig (Hrsg.): Anthropogene Einflüsse auf die Grundwasserbeschaffenheit in Niedersachsen; Band 34, 91-110; Braunschweig (1982)

Hengsberger, K. S.: Vergleich von Methoden zur Ermittlung der Schutzfunktion der Grundwasserüberdeckung an ausgewählten Standorten in Hessen. 81 S. Masterarbeit (2018, unveröffentl.)

Hölting, B., Haertlé, T., Hohberger, K.-H., Nachtigall, K.H., Villinger, E., Weinzierl, W., Wrobel, J.-P.: Konzept zur Ermittlung der Schutzfunktion der Grundwasserüberdeckung. - Geol. Jb., C63, 5-24; Hannover (1995)

LAWA: Vorarbeiten und Hinweise für die Berichterstattung an die Kommission sowie für die Aufstellung eines Bewirtschaftungsplanes.- Arbeitshilfe zur Umsetzung der EG-Wasserrahmenrichtlinie/ Teil 3: 122 S.; München (2003)

LAWA: Arbeitshilfe zur Umsetzung der EGWasserrahmenrichtlinie, Teil 3, 36-37: Charakterisierung der Deckschichten (2003)

Liebhold, M.: Bewertung der Verschmutzungsempfindlichkeit des Grundwassers im Untersuchungsgebiet Mecklenburg-Vorpommern, Deutschland. 132 S. Masterarbeit (2018, unveröffentl.)

Umweltbundesamt: Charakterisierung der Empfindlichkeit von Grundwasserkörpern; Forschungsbericht 29922 278; 118. S.; 19 Karten 1:200 000 als Anlagen; Berlin (2002)

Voigt, H.-J.: Hydrogeologisches Kartenwerk der Deutschen Demokratischen Republik, Nutzerrichtlinie für die Karte der Grundwassergefährdung, Karte 4.- Zentrales Geologisches Institut, 16 S., Berlin (1987)

U.S. National Research Council: Groundwater vulnerability assessment, contamination potential under conditions of uncertainty.- National Academy Press, Washington, p 210 (1993)

Zaporozec, A.: Groundwater contamination inventory - A methodical Guide-VI, Series on groundwater 2; UNESCO, Paris (2002)

B. Leßmann, Wiesbaden

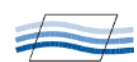




\section{Glosse}

\section{Coronakrisenmanagement - was alles schief lief}

Viele LeserInnen haben bei Grundwasser nachgefragt, ob Grundwasser (GW) nicht einmal erklären könnte, was denn da alles während der CoronaPandemie aus wassertechnischer Sicht schiefgelaufen ist. Wir konnten den aus einschlägigen Internetforen als Wasserblogger „Wasser-Egon“ bekannten Ernst-Egon Neggaawasser (EEN) aus der Nähe von Neckargmünd am Neckar für ein Interview gewinnen. Er hat sich nach eigenen Angaben seit mehreren Jahrzehnten mit Coronakrisen beschäftigt und gilt als Konifere unter den mittlerweile 1,5 Millionen Corona-Experten in Deutschland. Nachfolgend seine skalpellscharfe Analyse des Versagens der Bundesregierung beim Coronakrisenmanagement.

GW: Herr Neggaawasser, was sehen Sie als größten Fehler der Bundesregierung und der FH-DGGV, im Nachhinein, bei der Bewältigung der Corona-Krise?

EEN: Zunächst einmal, ich hätte alles anders gemacht. Ich hätte viel mehr von dem Impfstoff gekauft.

GW: Ja, welchen denn genau?

EEN: Ja, den Stoff mit der größten Wirkung natürlich und viel früher! Und, Heilandsack, dann hätte die FH-DGG hier mitwirken müssen, dass man da auch gleich eine Sonderregel schafft, dass man den Wirkstoff übers Trinkwasser hätte verteilen sollen lassen. Was hat Euer Präsident denn gemacht, nix!

GW: Wie bitte, übers Trinkwassersystem? Übers Leitungswasser?

EEN: Ja, genau, hätt' ich so gemacht. Diese ganze zeitfressende Diskussion, wer, wann oder wo nicht geimpft werden hätte sollen, das war sozialpolitisch komplett ungerecht. Ich hätt da die richtige Konzentration vom rich- tigen Anticoronastoff hineingemischt und dann hätt jeder so viel bekommen, wie er, reschpektive sie es braucht, direkt nach Haus, übers Trinkwasser. Ohne Nebenwirkungen am Arm!

GW: Interessante Idee, wie viel hätte man denn da reinmischen sollen?

EEN: Na so viel, dass es wirkt!!! Wirken muss es!!! Das ist immer das Wichtigste. Also, da stellen sich manche Politiker immer an und dann wundern sie sich, wenn die Leut' sich aufregen oder aufgeregt sind, wenn einfachste Lösungen nicht erkannt werden und angewandt werden.

GW: Die Menschen in Deutschland vertrauen uns Hydros, weil sie wissen, dass das Trinkwasser bestens untersucht und nach EU-Wasserrichtlinie „natürlich“ sein soll und es auch ist. Das wäre ein knallharter Paradigmenwechsel in der Wasserwelt. Wie hätten sie die unvermeidliche Aufregung in der Bevölkerung denn quasi wieder beruhigt nach so einem unglaublichen Eingriff in die Trinkwasserfreiheit?

EEN: Schon wieder so eine unpraktikable Frage! Natürlich wieder übers Leitungswasser, mit ein paar Beruhigungspülverchen. Alternativ hätte man Anticorona- und Beruhigungsmittel auch in kostenlosen Wasserflaschen abgeben können. In beiden Fällen hätte man immer das RKI und den Lauterbach als quasi beratende Impfstoffmischer natürlich zu Rate ziehen müssen, manchmal. Das wäre wieder sozialverträglich gewesen und jeder wäre wieder beruhigt gewesen. Dann wären die Leut auch vor lauter Beruhigung zuhaus geblieben und hätten nicht dauernd in den Innenstädten wilde Partys gefeiert.

GW: Wie wären Sie bei, zugegebenermaßen äußerst seltenen, Nebenwirkungen denn vorgegangen? Was hätten Sie der Bevölkerung gegeben? EEN: Ganz klare, eindeutige Antwort, kostenloses Sankt Leoganger Mineralwasser, kurz nach Mitternacht gezapft,
Ein Termin, den Sie sich merken sollten:

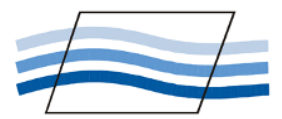

FH-DGGV-Fortbildung

Wasserhaushaltsuntersuchungen Grundlagen, Berechnungsmodelle, Anwendungsbeispiele Bad Soden-Salmünster

07.-08.04.2022

mit einem Schuss Nordschwarzwälder Himbeergeist, mindestens $65 \%$ ig. Hochwirksam gegen alles. Man hätte auch das Mineralwasser weglassen können.

GW: Mmh, zum Schluss noch kurz, wie würden Sie in Zukunft solche Krisen verhindern?

EEN: Einfach nicht mehr ausbrechen lassen, so ein gefährliches Tier!

GW: Danke Herr Neggaawasser für diese interessanten Einblicke in Wissen von Experten, die immer hinterher alles sehr genau vorhergesehen haben.

\section{Exkursionen}

\section{Der kleine Umweg - Die Siegfriedbrunnen in Grasellenbach und Heppenheim}

Liebe und Hass, Treue und Verrat, Güte und Gier, Krieg und Frieden, Mut und Arglist, Glück und Unglück sind der Stoff für Heldengeschichten, die die Menschheit seit jeher faszinieren. Eine solche Heldengeschichte ist die Nibelungensage, die als deutsches Nationalepos gilt, und sich wie das Drehbuch für eine Episode von Game of Thrones liest. Tatsächlich fußt die Nibelungensage auf historischen Ereig- 
nissen bzw. Persönlichkeiten zur Zeit der Völkerwanderung im 5. bis 7. Jahrhundert, die in der Folge dichterisch ausgeschmückt wurden. Zunächst wurde die Nibelungensage mündlich überliefert und in den folgenden Jahrhunderten niedergeschrieben, überarbeitet und erweitert. So ist es nicht verwunderlich, dass unterschiedliche Versionen entstanden. Die drei ältesten vollständig erhaltenen Handschriften $-\mathrm{A}$, B und $\mathrm{C}$ genannt - stammen aus dem 13. Jahrhundert. In allen Manuskripten spielt eine der Schlüsselszenen an einer Quelle und hat somit einen hydrogeologischen Bezug.

Siegfried, der Namensgeber der Quelle, ist ein edler Ritter und Erbe des Königreichs von Xanten. Er hatte einen Drachen getötet und war durch das Bad im Drachenblut unverwundbar geworden. Allerdings hatte beim Bad ein Lindenblatt seine Schulter bedeckt, sodass an einer kleinen Stelle seines Körpers die schützende Hornhaut fehlte. Zugleich konnte er durch den Einsatz magischer Waffen in den Besitz des riesigen Nibelungenschatzes kommen. Siegfried reist nach Worms, um am Königshof der mächtigen Burgunder um die Hand von Prinzessin Kriemhild anzuhalten. Diese denkt aber zunächst gar nicht ans Heiraten. Ihr Bruder, König Gunther, ist seinerseits auf Brautsuche. Gunther hat sich in den Kopf gesetzt, Brunhild, die Herrscherin Islands, zur Frau zu nehmen. Allerdings ist diese nur bereit einen Mann zu hei-

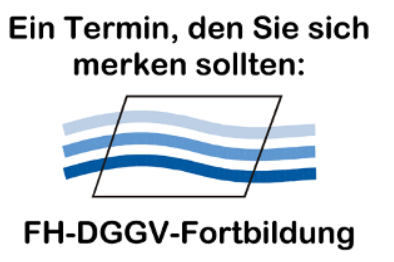

Angewandte Grundwassermodellierung I

Bad Soden-Salmünster

20.-23.04.2022 raten, der sie im Kampf besiegt, was aufgrund ihrer übernatürlichen Kräfte nahezu unmöglich ist. Siegfried hilft den Burgundern zunächst, einen Kriegszug der Sachsen und Dänen abzuwehren und reist dann mit Gunther, Hagen von Tronje und einem weiteren Gefolgsmann nach Island. Dort gelingt es Siegfried durch seine magische Tarnkappe unerkannt an Gunthers Seite zu kämpfen und Brunhild zu besiegen. Brunhild kann sich ihre Niederlage gegen den vermeintlich schwächeren Gunther nicht erklären, sodass Siegfried nach der Hochzeit in Worms in der Hochzeitsnacht erneut zu Gunthers Gunsten eingreifen muss. Erst nach dem Verlust der Jungfräulichkeit verliert Brunhild ihre übernatürlichen Kräfte. Kriemhild verliebt sich zwischenzeitlich in Siegfried, sodass beide ebenfalls heiraten und nach Xanten ziehen. Doch Brunhild geben die Ereignisse um ihre Heirat keine Ruhe und sie ahnt, dass Siegfried seine Hände im Spiel hatte. Auf ihre Bitte hin besuchen Kriemhild und Siegfried Jahre später Worms. Beide Königinnen streiten mehrfach heftig, Eitelkeiten werden verletzt. Hagen von Tronje möchte die Ehre seiner Königin verteidigen und argumentiert, dass durch die Beseitigung Siegfrieds auch der Nibelungenschatz gewonnen werden könnte. Schließlich wird mit Gunthers Zustimmung der Plan gefasst, Siegfried zu ermorden. Durch eine List gelingt es Hagen von Tronje Kriemhild zu entlocken, an welcher Stelle Siegfried verwundbar ist. Die Intrige beginnt mit einem Ausritt zur Jagd zum nahegelegenen Odenwald. Bei dem anschließenden Festmahl im Gelände fehlen allerdings die Getränke, denn Hagen von Tronje hatte die Diener absichtlich damit an einen falschen Ort geschickt. Um den Durst zu löschen, vereinbaren Siegfried und Hagen von Tronje einen Wettlauf zu einer nahegelegenen Quelle, den Siegfried unter erschwerten Bedingungen - er trug seine komplette Ausrüstung - gewinnt. An der Quelle angekommen wartet Sieg- fried bis König Gunther eingetroffen ist. Erst nachdem dieser getrunken hat, legt Siegfried seine Waffen ab, beugt sich vor und trinkt selbst aus der Quelle. In diesem Moment ergreift Hagen von Tronje dessen Speer und ermordet Siegfried hinterrücks mit seiner eigenen Waffe.

Aufgrund detaillierter geografischer Angaben ist davon auszugehen, dass die Verfasser oder Bearbeiter der Nibelungensage eine konkrete Vorstellung dieser Szene hatten und die Handlung an einer real existierenden Quelle verorteten. Doch aufgrund der verschiedenen Manuskripte und Unsicherheiten bei der Interpretation der geografischen Angaben werden etwa 10 verschiedene Quellen als Siegfriedbrunnen im Odenwald und angrenzenden Gebieten in Betracht gezogen.

Der bekannteste Siegfriedbrunnen - eigentlich müsste es Siegfriedquelle heißen - befindet sich östlich von Grasellenbach (Abb. 4) und geht auf den südhessischen Heimatforscher und Staatsdiener Johann Friedrich Knapp (1776-1848) zurück. Dieser verwies darauf, dass die Einheimischen diese Quelle eh und je Siegfriedbrunnen nannten, der Überlieferung nach dort ein Ritter namens Siegfried ermordet wurde und dementsprechend an der Quelle ein Sühnekreuz stünde. Er konnte Angaben in Handschrift C plausibel mit dieser Quelle in Verbindung bringen. Spehtsharte - in einigen modernen hochdeutschen Übertragungen fälschlich mit Spessart wiedergegeben - sei der oberhalb befindliche $548 \mathrm{~m}$ hohe Spessartskopf, Wasgenwald sei der Name des Waldes, der für den nahegelegenen Fluss und den Ort Weschnitz namensgebend sein könnte, und die Ortsbezeichnung Otenhaim könnte sich auf einen heute nicht mehr existierenden Weiler namens Dotenhan in der Gemarkung Grasellenbachs beziehen. Der Siegfriedbrunnen in Grasellenbach wird intensiv touristisch vermarktet. So führte der 2008 ausgewiesene - und mittlerweile erheblich erweiterte - Nibelungensteig 
ursprünglich auf gut $40 \mathrm{~km}$ von Zwingenberg an der Bergstraße bis an diesen Siegfriedbrunnen. Die Interpretation dieses Standortes als Siegfriedbrunnen hat allerdings zwei Schwächen: Hagen von Tronje wies die Diener an, den Wein auf den Spetsharte zu bringen. Wenn die Getränke tatsächlich nur wenige $100 \mathrm{~m}$ oberhalb der Quelle lagerten, dann hätte die Jagdgesellschaft gleich dorthin gehen können. Nach Einbruch der Dunkelheit ließ Hagen von Tronje Siegfrieds Leiche nach Worms bringen. Der Leichenzug kam laut Nibelungenlied noch vor Tagesanbruch an. Damit scheiden die konkurrierenden Lokationen im Odenwald eigentlich aus, denn die Entfernung für einen nächtlichen Ritt nebst Fährfahrt über den Rhein ist einfach zu groß.

Im Südwesten Heppenheims befindet sich mein persönlicher Favorit für die ,echte' Siegfriedquelle (Abb. 5). Die ursprünglich als Lindenbrunnen bezeichnete Quelle wurde infolge von Nachforschungen des Darmstädter Archivdirektor Julius Reinhard Dietrich 1931 in Siegfriedbrunnen umbenannt. Im Gegensatz zu allen anderen Siegfriedbrunnen befindet sich dieser nicht im sondern vor dem Odenwald, wie es eigentlich im Nibelungenlied heißt. Der Wasgenwald wäre der Wasenwald, ein unweit befindliches Sumpfgebiet, an der Weschnitz. Der Spehtsharte könnte als Spissert gedeutet werden, ein Waldgebiet bei Hüttenfeld, $7 \mathrm{~km}$ entfernt. Diese im Vergleich zu Grasellenbach größere Entfernung passt besser zu den Angaben im Nibelungenlied. Und Otenhaim wäre der benachbarte Lorscher Ortsteil Seehof, der einst von Uta von Calw ans Kloster Lorsch gestiftet wurde und im Mittelalter deswegen wohl Uotenheim hieß. Jedenfalls wird Uta von Calw in Handschrift C erwähnt. Überhaupt ist die Nähe Heppenheims zu dem einst bedeutenden Kloster Lorsch ein weiteres Argument, denn der Bearbeiter der Handschrift $\mathrm{C}$ lebte vermutlich in dem dortigen Kloster.

Abb. 4 Der Siegfriedbrunnen östlich von Grasellenbach entsprang einst dem Unteren Buntsandstein

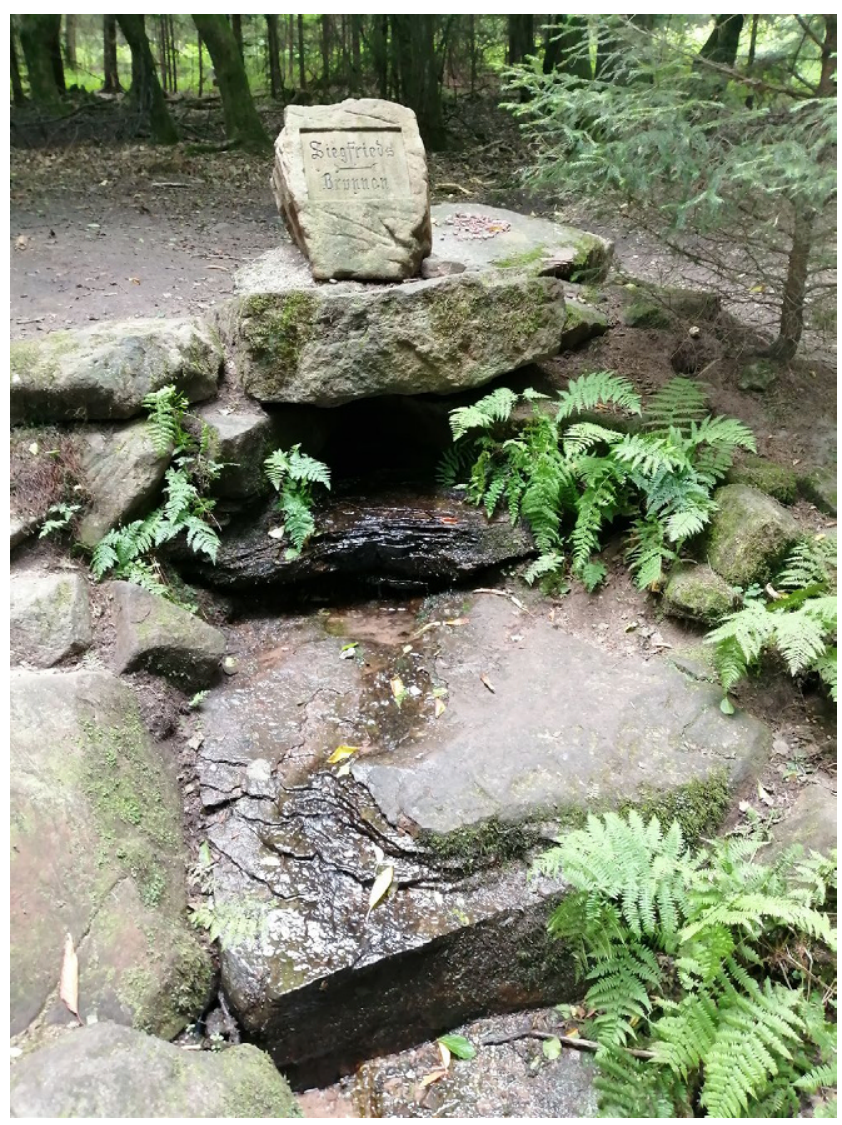

Der Siegfriedbrunnen der Nibelungensage ist ein schönes Beispiel dafür, dass Quellen für Menschen in der Antike und mit Mittelalter als Spenderinnen frischen und sauberen Trinkwassers eine bedeutende Rolle hatten. Nicht umsonst wurden einige Quellen als heilige Orte verehrt, Siedlungen an ihnen gegründet und hatten Quellen in der Mythologie eine besondere Bedeutung. Umso bedauerlicher ist es, wie sich diese natürlichen Ressourcen durch das Handeln des Menschen in den vergangenen Jahrzehnten verändert haben. Die Siegfriedquelle in Grasellenbach versiegte in den 50er Jahren, nachdem der umliegende Wald aus forstwirtschaftlichen Gründen von einem Laubwald in einen Nadelwald verwandelt wurde und sich dadurch die Grundwasserverhältnisse änderten. Heute wird der Siegfriedbrunnen aus dem öffentlichen Wassernetz gespeist und somit eine Quelle vorgetäuscht. Durch Ausdehnung
Heppenheims nach Westen nach dem Zweiten Weltkrieg führten Bachregulierungen und die Anlage von Entwässerungsgräben zu einer Grundwasserabsenkung von insgesamt etwa zwei Metern. Zunächst versiegte die Quelle und später fiel der Brunnen an derselben Stelle trocken. Heute befindet sich die einstiege Quelle in einem dicht bebauten Wohn- und Gewerbegebiet.

Anreise: Mit dem PKW auf der A5 Darmstadt-Heidelberg die Ausfahrt 31 „Heppenheim“ nehmen und der B460 stadteinwärts nach Osten folgen. Die B460 ist passenderweise zugleich die Touristikroute Siegfriedstraße. An der großen Kreuzung am Ortseingang rechts in die Tiergartenstraße einbiegen. Der Siegfriedbrunnen befindet sich nach etwa einem Kilometer in einem kleinen, unscheinbaren Park auf der linken Seite. Der B460 dann etwas mehr als $20 \mathrm{~km}$ Richtung Erbach folgen. Hinter Weschnitz befindet sich auf einer Anhöhe eine Kreuzung mit 


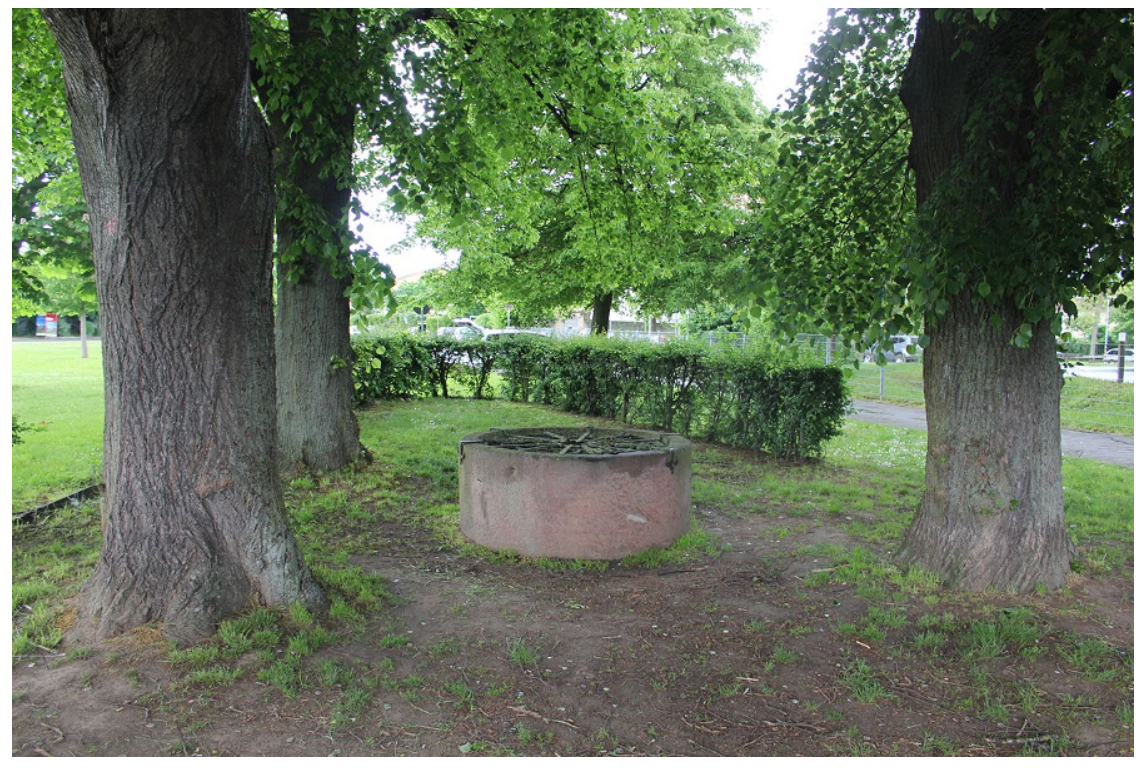

Abb. 5 Der Siegfriedbrunnen in Heppenheim befindet sich in einem kleinen Park, eingerahmt von drei Linden

einer Landstraße - der nahegelegene Kahlberg markiert übrigens den Tripelpunkt der Einzugsgebiete von Rhein, Main und Neckar. An der Kreuzung dann rechts in die L3105 nach Grasellenbach abbiegen. In der Ortsmitte dann links in die Güttersbacher Straße abbiegen und der Straße bis zum Ende folgen, wo sich ein Wanderparkplatz befindet. Von hier aus kann die Siegfriedquelle entweder auf einem breiten Forstweg mit geringer Steigung oder entlang eines etwas stei- leren, aber direkteren Wanderweges nach 25 bis 40 Minuten erreicht werden. Auf dem Rückweg kann an einer Grillhütte gepicknickt oder in einem Kneippbecken eine Erfrischung eingeholt werden. Vom ICE/IC-Bahnhof Bensheim oder dem RE/RB-Bahnhof Heppenheim (Bahnlinie FrankfurtDarmstadt-Heidelberg/Mannheim) gibt es einige Nahverkehrsbuslinien in den Odenwald. Sehenswert und zu Fuß oder mit dem Fahrrad erkundbar sind die Heppenheimer Altstadt sowie das UNSECO Weltkulturerbe Kloster Lorsch. Auch kann vom RE/RB-Bahnhof Zwingenberg der Nibelungensteig in Angriff genommen werden. Weitere Sehenswürdigkeiten des nationalen Geoparks Bergstraße-Odenwald sind das Felsenmeer bei Reichenbach, das Drachenmuseum in Lindenfels, sowie das Auerbacher Schloss, von dem man einen wunderbaren landschaftlichen Überblick des nördlichen Oberrheingrabens erhält und bei guten Sichtverhältnissen bis in den Taunus, den Donnersberg in der gegenüberliegenden Pfalz sowie Mannheim blicken kann.

\section{Literatur}

Becht, A., Diehl, M., Friedrich, R., Fritsche, J.-G., Hergesell, M., Hoselmann, C., Hug-Diegel, N., Kämmerer, D., Kött, A., Leßmann, B., Lorenz-Hefner, C., Ludwig, F., Marold, U., Nesbor, H.-D., von Pape, W.-P., Pöschl, W., Prein, A., Rückert, H., Schneider, M.W., Senner, R., Vero, G.: Hydrogeologie von Hessen - Odenwald und Sprendlinger Horst. Grundwasser in Hessen, Heft 2. HLNUG, Wiesbaden, 136 Seiten (2017)

Offizielle Webseite Geonaturpark BergstraBe-Odenwald: https://geo-naturpark.net/

R. Schäffer, Bensheim

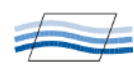




\section{Veranstaltungskalender}

\section{Tagungen und Kongresse}

\section{Paris, Frankreich \\ 06.-08.12.2021}

IAH: ISARM 2021 - 2nd International Conference on Transboundary Aquifers Internet: https://en.unesco.org/ conference/isarm 2021

\section{Lübeck}

\section{9.-11.03.2022}

ITVA: Altlastensymposium 2022

Internet: www.itv-altlasten.de

\section{Nikosia, Zypern}

\section{0.-22.03.2022}

IAH: 12th International Hydrogeological Conference

Internet:

https://hydrogeologyconference 2020 .com.cy/

\section{Jena}

23.-26.03.2022

28. FH-DGGV-Tagung: Grundwasser - Klima - Gesellschaft

Internet: www.fh-dggv.de/jena-2022

\section{Augsburg}

27.-29.04.2022

19. Deutsches Talsperrensymposium Internet: www.talsperrensymposium.de

\section{Caserta, Italien}

26.-30.06.2022

IAH: MinWat2020 - 3rd International multidisciplinary conference on mineral and thermal waters. (Postponed from 2020)

Internet: www.minwatitaly2020.org

\section{Fortbildungslehrgänge}

\section{FH-DGGV}

Telefon: +49 6321 484-784

Telefax: +49 6321 484-783

E-Mail: geschaeftstelle@fh-dggv.de Internet: www.fh-dggv.de

\section{5.-26.02.2022}

Durchführung und Auswertung von Pumpversuchen in Bad Soden-Salmünster

\section{3.-04.03.2022}

Beschaffenheit des Grundwassers in Bad Soden-Salmünster

\section{5.-17.03.2022}

Hydrogeochemische Systeme quantifizieren mit PHREEQC in Hildesheim

\subsubsection{2}

Bohrlochgeophysik in der Praxis - vom Messwert zum Ergebnis in Jena

\subsubsection{2}

Neue Technologien zur Erforschung der unsichtbaren Welt von aquatischen Lebensräumen in Jena

\section{7.-08.04.2022}

Wasserhaushaltsuntersuchungen Grundlagen, Berechnungsmodelle, Anwendungsbeispiele in Bad Soden-Salmünster

\section{0.-23.04.2022}

Angewandte Grundwassermodellierung I: Einführung in die Strömungs- und Transportmodellierung mit praktischen Anwendungen am $\mathrm{PC}$ in Bad Soden-Salmünster
16.-17.05.2022

Grundwasserversalzung - Probleme, Methoden und Lösungsansätze in Bad Soden-Salmünster

\section{5.-28.05.2022}

OpenSource GIS und Datenbanken für die hydrogeologische Praxis in Bad Soden-Salmünster

\section{7.-08.10.2022}

Durchführung und Auswertung von Pumpversuchen in Bad Soden-Salmünster

\section{2.-05.11.2022}

Angewandte Grundwassermodellierung II - Komplexe Aquifersysteme: Strömungs- und Transportmodellierung für Fortgeschrittene mit praktischen Anwendungen am $\mathrm{PC}$ in Bad Soden-Salmünster

\section{DGFZ e. V.}

Telefon: +49 351 4050-676

Telefax: +49 351 4050-679

E-Mail: weiterbildung@dgfz.de Internet: www.dgfz.de

\subsubsection{2}

Probennahme von Abfällen nach LAGA PN98

\subsubsection{2}

Ausbau und Benutzung von Gewässern

\section{7.-19.05.2022}

Qualifizierung von Sachverständigen für Bodenschutz und Altlasten, Kompaktseminar gem. $\S 18$ BBodSchG bzw. $\$ 36$ GewO

01.-02.06.2022

Grundwasserneubildung

05.-07.10.2022

QGIS in der Hydro(geo)logie 


\section{Veranstaltungs- ankündigungen}

\section{FH-DGGV bei der GeoTHERM 2022, 17.-18.02.2022}

Am 17. und 18. Februar 2022 findet in Offenburg zum fünfzehnten Mal die „GeoTHERM expo \& congress“ statt.

Die FH-DGGV wird als Aussteller vertreten sein und über aktuelle Veröffentlichungen, die Zeitschrift Grundwasser, Fortbildungsveranstaltungen und Tagungen der FH-DGGV informieren.
Außerdem besteht die Möglichkeit, Mitgliedsangelegenheiten, wie z.B. Adressenänderungen und Aufnahmeanträge vor Ort ohne großen Aufwand vorzunehmen.

Weitere Informationen zur GeoTHERM expo \& congress: www.geotherm-offenburg.de

Dr. Maike Rüsgen

E-Mail: maike.ruesgen@fh-dggv.de
Hinweis des Verlags Der Verlag bleibt in Hinblick auf geografische Zuordnungen und Gebietsbezeichnungen in veröffentlichten Karten und Institutsadressen neutral. 


\section{Jahrestagung der FH-DGGV Grundwasser - Klima - Gesellschaft} 23.-26. März 2022 in Jena

7 FH-DGGV

\section{Sessions}

- Grundwasserressourcen und Ökosysteme in Karstregionen

- Identifizierung landwirtschaftlich bedingter Indikatoren im Grundwasser

- Grundwasserversalzung - Ursachen, Herausforderungen und Ausblicke

- Hydrogeologie arider Gebiete

- Hydrogeologie in der Praxis I - Fallbeispiele der Staatlichen Geologischen Dienste

- Hydrogeologie in der Praxis II

- Künstliche Intelligenz in der Hydrogeologie

- Temperaturänderungen im Grundwasser: Ursachen, Prozesse und Auswirkungen

- Bergbau und Grundwasser

- Geotechnische Anwendungen in Grundwassersystemen

- Verweilzeiterkundung in Grundwasserleitern mittels Tracern und Grundwasseraltern

- Dürreperioden - Herausforderungen für die zukünftige Grundwasserbewirtschaftung

- Artificial and natural groundwater recharge (IAH Session)

- Hydrogeologie und Standortauswahl für ein tiefengeologisches Endlager (mit DGGV)

- Grundwasserqualitätsentwicklung - Erkenntnisse aus Langzeitstudien in der Kritischen Zone

- Freie Themen

Vorläufiges Programm

23.03.2022

- Fortbildungsveranstaltungen I und II

- Exkursion I

- Forum Junge Hydrogeologen

- Jobbörse

- Icebreaker-Party

24.03.2022

- Vortrags- und Postersessions

- Mitgliederversammlung der FH-DGGV

- Gesellschaftsabend

25.03.2022

- Vortrags- und Postersessions

- Mitgliederversammlung der IAH

- Abendvortrag

26.03.2022

- Vortrags- und Postersessions

- Exkursionen II bis V

\section{Fortbildungsveranstaltungen \& Exkursionen}

Fortbildung I: Bohrloch-Geophysik in der Praxis - vom Messwert zum Ergebnis; Th. Woniket al.

Fortbildung II: Neue Technologien zur Erforschung der unsichtbaren Welt von aquatischen Lebensräumen; K. Küsel et al.

Exkursion I: Sanierung der industriellen Absetzanlagen der Wismut GmbH am Standort Seelingstädt; Leitung Dipl.-Geol. Ulf Barnekow

Exkursion II: Hainich-Exploratorium (Hainich Critical Zone Exploratory); Leitung: FSU Jena \& Nationalpark Hainich

Exkursion III: Salinares Tiefenwasser, Solegewinnung und Subrosion in Bad Frankenhausen und Umgebung; Leitung: Dr. Jörn Geletneky, Annett Peters (TLUBN)

Exkursion IV: Wald - Wasser - Wandel: Bedeutung und Vulnerabilität von Wald-/Forst-Grundwassereinzugsgebieten; Leitung: FSU Jena \& Thüringen Forst

Exkursion V: Tiefbrunnen und Wasserwerk für die Trinkwasserversorgung Jena; Leitung: Dipl. Ing. Christoph Wittich

\section{Forum Junge Hydrogeologen}

Studierenden und Promovierenden wird die Möglichkeit gegeben, in einer gesonderten Veranstaltung interessante Studienprojekte, M.Sc.- bzw. Doktorarbeiten vorzustellen. Der beste Vortrag im Forum Junger Hydrogeologen wird mit einer Teilnahme an einer FH-DGGV-Fortbildungsveranstaltung im Jahr 2022/23 honoriert.

\section{Kontaktadressen}

Geschäftsstelle der FH-DGGV

Dr. R. Kaufmann-Knoke

E-Mail: geschaeftsstelle@fh-dggv.de

Friedrich-Schiller-Universität Jena

Regina Piechnick

E-Mail: regina.piechnick@uni-jena.de

Internet: www.fh-dggv.de/jena-2022 


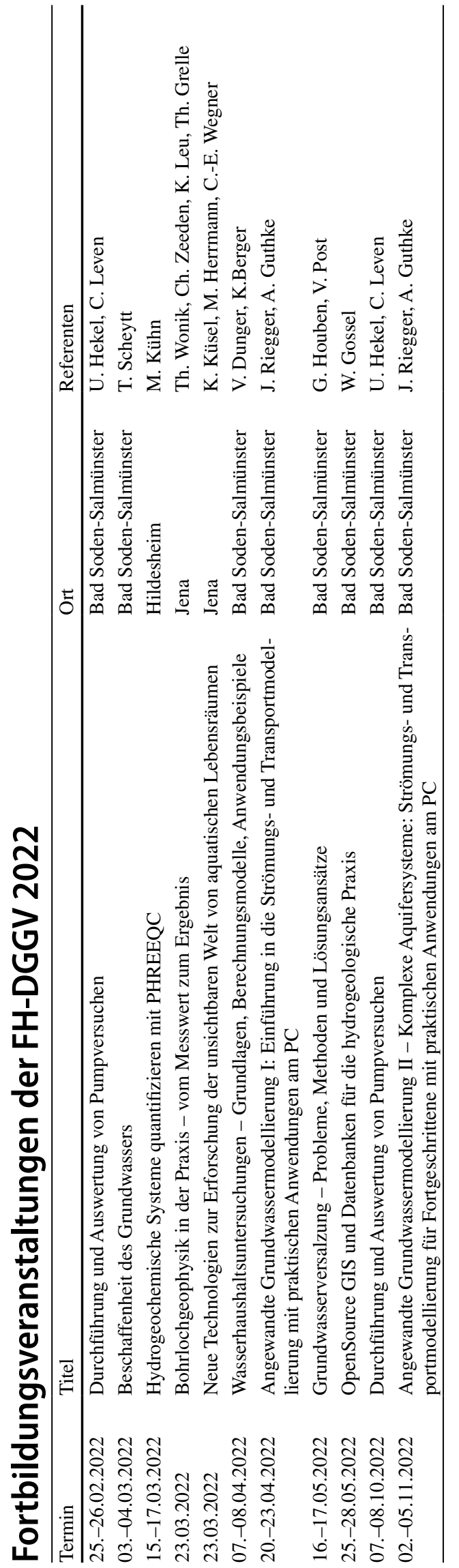

